\title{
Optimal Scheduling of a Microgrid Including Pump Scheduling and Network Constraints
}

\author{
Ashok Krishnan $\mathbb{D}^{1}{ }^{1}$ L. P. M. I. Sampath, ${ }^{2}$ Y. S. Foo Eddy $\mathbb{D}^{1},{ }^{1}$ and H. B. Gooi ${ }^{1}$ \\ ${ }^{1}$ School of Electrical and Electronic Engineering, Nanyang Technological University, 50 Nanyang Avenue, Singapore 639798, Singapore \\ ${ }^{2}$ Interdisciplinary Graduate School, Nanyang Technological University, 21 Nanyang Link, Singapore 637371, Singapore
}

Correspondence should be addressed to Ashok Krishnan; ashok004@e.ntu.edu.sg

Received 26 January 2018; Accepted 3 April 2018; Published 10 July 2018

Academic Editor: João Soares

Copyright (C) 2018 Ashok Krishnan et al. This is an open access article distributed under the Creative Commons Attribution License, which permits unrestricted use, distribution, and reproduction in any medium, provided the original work is properly cited.

\begin{abstract}
This paper proposes an efficient energy management system (EMS) for industrial microgrids (MGs). Many industries deploy large pumps for their processes. Oftentimes, such pumps are operated during hours of peak electricity prices. A lot of industries use a mix of captive generation and imported utility electricity to meet their energy requirements. The MG considered in this paper includes diesel generators, battery energy storage systems, renewable energy sources, flexible loads, and interruptible loads. Pump loads found in shipyard dry docks are modelled as exemplar flexible industrial loads. The proposed EMS has a two-stage architecture. An optimal MG scheduling problem including pump scheduling and curtailment of interruptible loads (ILs) is formulated and solved in the first stage. An optimal power flow problem is solved in the second stage to verify the feasibility of the MG schedule with the network constraints. An iterative procedure is used to coordinate the two EMS stages. Multiple case studies are used to demonstrate the utility of the proposed EMS. The case studies highlight the efficacy of load management strategies such as pump scheduling and curtailment of ILs in reducing the total electricity cost of the MG.
\end{abstract}

\section{Introduction}

Industrial power networks may comprise distributed generators, battery energy storage systems (BESSs), and different types of loads. As such, industrial power networks can be treated as grid-connected MGs. An EMS facilitates efficient MG operation by minimizing the overall electricity cost. The EMS determines an optimal schedule and dispatch for each distributed generator, BESS, flexible load, and interruptible load (IL) in the MG while respecting various technical and operational constraints. The EMS is also capable of incorporating load management strategies in its optimal scheduling problem formulation. Furthermore, the EMS needs to consider power flow equations and system security constraints while optimally dispatching the MG components. This is considered in an optimal power flow (OPF) problem incorporated in the EMS architecture. Thus, formulating an efficient energy management (EM) problem is a complex task due to the need to integrate both unit commitment (UC) and OPF problems. This normally results in the EM problem being formulated by ignoring network losses and system security constraints. The EM problem then simplifies to a UC problem which is usually formulated as a mixed integer linear programming (MILP) or mixed integer quadratic programming (MIQP) problem. However, the feasibility of results obtained using such formulations is questionable owing to potential violations of the network constraints and the absence of power losses in the formulation.

In Singapore, the wholesale electricity prices are updated every 30 minutes. Large industrial customers have the option of sourcing their electricity requirements directly from the wholesale electricity market through SP Services Limited. In this scenario, industries need to contend with the risk of increased costs due to volatile electricity prices. The contestable consumers' electricity bills usually comprise two segments: (i) energy charge and (ii) capacity charge. The energy charge is calculated by taking the product of electricity charge and energy consumed. The capacity charge is computed on a monthly basis. The capacity charge is calculated by taking the product of contracted capacity and contracted capacity price. A huge uncontracted capacity charge is incurred if the maximum power demand exceeds the contracted capacity at 
any point of time. Large pumps constitute a major portion of the electrical load demand in many industrial facilities. Due to the large pumping requirements in many industries, pump capacities are typically in the region of several MWs. In other words, pump usage has a significant bearing on the maximum load demand in many industrial facilities. Pump usage is also constrained by operational requirements in many industries whereby a certain quantity of water or other liquid needs to be pumped within a defined time frame. Optimal planning and scheduling of pump usage along with timely curtailment of ILs can help in lowering the maximum demand of the industrial facility, thereby leading to a reduction in the electricity cost.

With the advent of deregulated power systems, load management strategies have recently attracted wide research interest. A recent survey of existing demand response models and approaches can be found in [1]. Load management strategies have been proposed for rural water pumping stations [2] and flour mills [3, 4] among other applications. An optimal pump scheduling problem formulation for a rural two-stage water pumping station was developed in [2]. A generic optimal industrial load management scheme compatible with energy hub management systems was developed in [3] for process scheduling in industries such as flour mills and water pumping facilities. The mixed logical dynamical (MLD) approach was used to formulate and solve an optimal scheduling problem for district heating networks including generation sources, thermal energy storage systems, and flexible thermal loads in [5]. A load management strategy meeting objectives such as maximum user comfort or minimum cost for thermostatically controlled household appliances was presented in [6]. A recent work also developed an EMS for district heating networks including flexible loads while ensuring adequate user comfort at minimal cost [7].

Development of EMSs for MGs has been an active research area in recent years. In this context, model predictive control (MPC) based schemes have been widely adopted in recent years for power system scheduling applications. A few typical examples of such schemes can be found in [810]. However, as mentioned earlier, most of these schemes including $[8,9]$ do not account for network constraints due to the difficulties involved in solving the optimization problem. In other words, most of these schemes consider the UC and OPF problems separately. A few works have integrated the $\mathrm{UC}$ and OPF problems. The authors of [11] integrated the UC and OPF problems for a conventional power system without considering the presence of BESSs, renewable energy sources (RESs), and ILs. More recent works such as [12, 13] consider centralized cooperative schemes for standalone MGs. A centralized controller addresses the power sharing problem among interconnected MGs in [14]. The authors of [15] proposed a jump and shift method for integrating the UC and OPF problems while including BESSs and RESs in the network. This approach was adapted for a multimicrogrid scenario in the authors' recent work [16]. However, these works do not consider load management strategies in the problem formulation.

In this paper, comprehensive, component wise models of exemplar MGs are first developed. The MGs modelled in this paper comprise diesel generators (DGs), BESSs, RESs, flexible pump loads, and ILs. The MLD framework is used to model the DGs, BESSs, and ILs. Subsequently, an EMS is proposed for optimally scheduling the MG. The proposed EMS has a two-stage architecture along the lines of [15] to minimize operational costs while ensuring that the network constraints are not violated. The proposed EMS also incorporates efficient load management strategies such as pump scheduling and curtailment of ILs. The pump scheduling scheme is formulated to save electricity costs while respecting certain operational constraints. The incorporation of load management strategies such as pump scheduling into the overall two-stage MG EMS architecture is the key contribution of this paper. This result is a new optimal MG scheduling problem formulation which helps in reducing the overall cost of operation for the MG while respecting various technical and operational constraints. The efficacy of the proposed EMS is demonstrated through case studies under different operational scenarios. The case studies also demonstrate the effectiveness of including ILs in the overall optimal scheduling problem.

The remainder of this paper is organized as follows: Section 2 develops first principle models of all the MG components. Section 3 describes the formulation of the EM problem. An overview of the iterative solution approach implemented in the EMS is also presented in Section 3. Section 4 presents numerical results obtained from different case studies performed to demonstrate the efficacy of the proposed optimization model. Section 5 provides some concluding remarks and directions for future work.

\section{Industrial Microgrid Model}

This section develops first principle models of the DGs, BESSs, RESs, flexible pump loads, and ILs considered in this paper.

2.1. Diesel Generators. DGs are controllable in nature. The fuel cost of DG $f$ is determined by a quadratic function of its real power output as shown below [15]. Furthermore, start-up costs are imposed on the DGs. Minimum uptime (UT) and downtime (DT) constraints also restrict the operation of the DGs.

$$
C_{\mathrm{DG}}=\sum_{\substack{k \in \mathscr{K} \\ f \in \mathscr{F}}}\left(b_{\mathrm{SU}, k}^{f} C_{\mathrm{SU}}^{f}+b_{\mathrm{DG}, k}^{f}\left(c_{0}^{f}+c_{1}^{f} P_{\mathrm{DG}, k}^{f}+c_{2}^{f}\left(P_{\mathrm{DG}, k}^{f}\right)^{2}\right)\right) .
$$

In (1), the first term represents the start-up cost while the second term represents the fuel cost of the corresponding DG. All the technical parameters of the 3 DGs considered in this paper are provided in Table 1.

2.2. Battery Energy Storage Systems. In this work, a realistic BESS model is considered including intertemporal constraints on the state of charge (SOC). A battery degradation cost function accounts for the impact of charging and 
TABLE 1: Technical parameters of the DGs in the MGs.

\begin{tabular}{lccccccc}
\hline $\begin{array}{l}\mathrm{DG} \\
\#\end{array}$ & $\begin{array}{c}c_{0}^{f} \\
(\$)\end{array}$ & $\begin{array}{c}c_{1}^{f} \\
(\$ / \mathrm{MW})\end{array}$ & $\begin{array}{c}c_{2}^{f} \\
\left(\$ / \mathrm{MW}^{2}\right)\end{array}$ & $\begin{array}{c}\min P_{\mathrm{DG}, k}^{f} \\
(\mathrm{MW})\end{array}$ & $\begin{array}{c}\max P_{\mathrm{DG}, k}^{f} \\
(\mathrm{MW})\end{array}$ & $\begin{array}{c}C_{\mathrm{SU}}^{f} \\
(\$)\end{array}$ & $\begin{array}{c}\mathrm{min} \mathrm{UT} / \mathrm{DT} \\
(\mathrm{h})\end{array}$ \\
\hline 1 & 80 & 30 & 1 & 0.1 & 3 & 3 & 30 \\
2 & 200 & 60 & 2 & 0.1 & 3 & 3 & 3 \\
3 & 1000 & 50 & 3 & 0.1 & 3 & 5 & 3 \\
\hline
\end{tabular}

discharging events on the cost of purchasing the BESS. The overall BESS model is presented below [17].

$$
\begin{aligned}
\operatorname{SOC}_{k+1}^{e} & =\operatorname{SOC}_{k}^{e}+\frac{\left(\eta_{\mathrm{c}}^{e} P_{\mathrm{bc}, k}^{e}-P_{\mathrm{bd}, k}^{e} / \eta_{\mathrm{d}}^{e}\right)}{P_{1 \mathrm{C}}^{e}}, \\
& \forall k \in \mathscr{K}, \forall e \in \mathscr{E} \\
\mathrm{SOC}_{\text {min }}^{e} & \leq \mathrm{SOC}_{k+1}^{e} \leq \operatorname{SOC}_{\text {max }}^{e}, \quad \forall k \in \mathscr{K}, \forall e \in \mathscr{E} \\
0 & \leq P_{\mathrm{bc}, k}^{e} \leq P_{\mathrm{bc}, \text { max }}^{e}, \quad \forall k \in \mathscr{K}, \forall e \in \mathscr{E} \\
0 & \leq P_{\mathrm{bd}, k}^{e} \leq P_{\mathrm{bd}, \text { max }}^{\mathrm{e}}, \quad \forall k \in \mathscr{K}, \forall e \in \mathscr{E} .
\end{aligned}
$$

The evolution of the BESS SOC is described by (2). The SOC evolution depends on the charging and discharging powers and the charging and discharging efficiencies. A binary variable is used to distinguish between the charging and discharging events to prevent simultaneous charging and discharging. Interested readers may refer to [8] for more details. Intertemporal constraints on the SOC evolution are expressed in (3) while the bounds on charging and discharging powers are expressed in (4) and (5), respectively. The overall BESS cost function is expressed as follows:

$$
C_{\mathrm{BESS}}=\sum_{\substack{k \in \mathscr{K} \\ e \in \mathscr{C}}} \frac{I^{e}}{2 B_{\mathrm{cap}}^{e} N^{e}}\left(\frac{P_{\mathrm{bc}, k}^{e}}{T_{\mathrm{bc}}^{e}}+\frac{P_{\mathrm{bd}, k}^{e}}{T_{\mathrm{bd}}^{e}}\right) .
$$

In this paper, all the BESSs are considered to have the following parameters: $P_{\mathrm{bc}, \max }^{e}=P_{\mathrm{bd}, \max }^{e}=300 \mathrm{~kW}, B_{\mathrm{cap}}^{e}=$ $1020 \mathrm{kWh}, N^{e}=6000 \mathrm{~h}, P_{1 \mathrm{C}}^{e}=1020 \mathrm{~kW}, \mathrm{SOC}_{\min }=0.2, \eta_{\mathrm{c}}^{e}=\eta_{\mathrm{d}}^{e}$ $=0.95, \mathrm{SOC}_{\max }=0.9$, and $I^{e}=\$ 408,000$. Furthermore, BESS operation is constrained by the following $[18,19]$ :

$$
\mathrm{SOC}_{1}^{e}=\mathrm{SOC}_{25}^{e}, \quad \forall e \in \mathscr{E} .
$$

In this work, the load profiles used for the MG scheduling problems were adapted from the IEEE RTS-96 load profiles provided in [20]. As such, in many cases, the system load profile may not differ significantly between two consecutive days. In this scenario, it is important to ensure that the flexibility provided by the BESS scheduling is available every day to the system operator. To provide this flexibility, the initial and final SOCs of the BESSs are constrained to be equal as shown in (7).

2.3. Renewable Energy Sources. Renewable energy sources such as solar photovoltaic (PV) and wind power plants are included as components of the MGs which are modelled in this paper. The operating costs of these RESs are assumed to be 0 [16]. The following paragraphs describe the modelling of the solar PV and wind power plants.

The power output from the wind power plant is proportional to the cube of the wind velocity and is calculated using the following equation:

$$
P_{\text {wind }}=0.5 C_{\mathrm{p}} k \rho A\left(v_{\text {wind }}\right)^{3} .
$$

Among other PV performance models, the five-parameter array performance model has been extensively used by researchers [21]. The PV performance model is used to extract the current-voltage $(I-V)$ curve and the maximum power point (MPP) which can help in improving the performance of the PV system. The steady-state PV module performance is described as follows [22]:

$$
\begin{aligned}
I_{\mathrm{L}} & -I_{\mathrm{S}}\left\{\exp \left[\alpha\left(v_{\mathrm{pv}}+R_{\mathrm{S}} i_{\mathrm{pv}}\right)\right]-1\right\}-\frac{v_{\mathrm{pv}}+R_{\mathrm{S}} i_{\mathrm{pv}}}{R_{\mathrm{Sh}}}-i_{\mathrm{pv}} \\
\quad & =0 \\
P_{\mathrm{pv}} & =v_{\mathrm{pv}} i_{\mathrm{pv}},
\end{aligned}
$$

where $\alpha=q / n_{\mathrm{s}} k T$ is the ideality factor. Furthermore, $k=$ $1.38 \times 10^{23} \mathrm{~J} / \mathrm{K}$ is the Boltzmann's constant, $q=1.6022 \times 10^{19}$ is the electronic charge, $K=298 \mathrm{~K}$ is the temperature, and $n_{\mathrm{s}}$ is the number of cells in series. Interested readers may refer to [22] and the references therein for further details about the solar PV and wind power plant models. In this work, the solar PV and wind power plant generation forecasts were obtained from [23]. In this paper, $P_{\mathrm{RES}, k}^{z}$ is used to represent the electrical power produced by RES $z$ (either $P_{\mathrm{pv}}$ or $P_{\text {wind }}$ ) during hour $k$.

2.4. Interruptible Loads. These are loads in the MG which are considered to be less important. ILs provide the EMS with a lot of flexibility while scheduling the MG components. The ILs may be shed if sufficient monetary compensation is paid. The total cost associated with the curtailment of all the ILs in a MG is calculated as follows [24]:

$$
C_{\mathrm{IL}}=\sum_{\substack{k \in \mathscr{K} \\ h \in \mathscr{H}}} p_{k}^{h} b_{\mathrm{IL}, k}^{h} P_{\mathrm{IL}, k}^{h} .
$$

Three ILs (IL 1, IL 2, and IL 3) are considered to be a part of the MGs modelled in this paper. The curtailment of each IL is constrained by the following:

$$
\begin{aligned}
0 & \leq P_{\mathrm{IL}, k}^{h} \leq 0.4 \mathrm{MWh}, \quad \forall k \in \mathscr{K}, \forall h \in \mathscr{H} \\
\sum_{k \in \mathscr{K}} P_{\mathrm{IL}, k}^{h} & \leq 2 \mathrm{MWh}, \quad \forall h \in \mathscr{H} .
\end{aligned}
$$


The constraints in (11) and (12) basically restrain the EMS from indiscriminately using the ILs to relax the scheduling problem. Equation (11) imposes an hourly constraint while (12) imposes a daily limit on the curtailment of individual ILs.

2.5. Pump Loads. Pump loads found in shipyard dry docks have been used as exemplar industrial loads in this paper. The power consumed by pump $m$ during hour $k, P_{\text {Pump }, k}^{m}$, is defined as follows [25]:

$$
P_{\text {Pump }, k}^{m}=C^{m} b_{k}^{m}, \quad \forall k \in \mathscr{K}, \forall m \in \mathscr{M} .
$$

Equation (13) implies that all the pumps run at fixed speeds and operate at rated power if scheduled. The constraints associated with the operation of the pumps are described in the following paragraphs.

Pumps are required to pump a certain amount of water or other liquid within the specified optimization period. This constraint is expressed as follows:

$$
\sum_{\substack{k \in \mathscr{K} \\ m \in \mathscr{M}}} Q^{m} \tau * b_{k}^{m} \geq V_{\mathrm{d}} .
$$

Large pumps cannot be started up or shutdown too frequently during the optimization period due to their large inertias. This constraint is expressed as follows:

$$
\begin{aligned}
\sum_{k \in \mathscr{K}} b_{\mathrm{SU}, k}^{m} & \geq b_{\mathrm{SU}, \max }^{m}, \quad \forall m \in \mathscr{M} \\
b_{\mathrm{SU}, k}^{m} & =b_{k}^{m}\left(b_{k}^{m}-b_{k-1}^{m}\right), \quad \forall k \in \mathscr{K}, \forall m \in \mathscr{M} .
\end{aligned}
$$

Equation (16) is linearized as follows [26]:

$$
\begin{aligned}
& b_{\mathrm{SU}, k}^{m} \leq \frac{\left(b_{k}^{m}+1-b_{k-1}^{m}\right)}{2}, \quad \forall k \in \mathscr{K}, \forall m \in \mathscr{M} \\
& b_{\mathrm{SU}, k}^{m} \geq \frac{\left(b_{k}^{m}-b_{k-1}^{m}\right)}{2}, \quad \forall k \in \mathscr{K}, \forall m \in \mathscr{M} .
\end{aligned}
$$

This work considers a total of 7 pumps including 3 main pumps and 4 auxiliary pumps. For the main pumps: $Q^{m}=$ $24,000 \mathrm{~m}^{3} / \mathrm{h}, C^{m}=1.45 \mathrm{MW}$, and $b_{\mathrm{SU}, \max }^{m}=1$. For the auxiliary pumps: $Q^{m}=1,200 \mathrm{~m}^{3} / \mathrm{h}, C^{m}=0.11 \mathrm{MW}$ and $b_{\mathrm{SU} \text {, } m a x}^{m}=10$. Finally, $V_{d}=200,000 \mathrm{~m}^{3}$, and $\tau=1 \mathrm{~h}$. It may be pertinent to mention here that despite the greater flexibility offered by the auxiliary pumps, they are inefficient compared to the main pumps. As a result, the advantage offered by the greater flexibility of the auxiliary pumps is diminished to a certain extent.

2.6. MLD Modelling Approach. The MLD formalism is a class of hybrid dynamical systems which has been used by several researchers for formulating power system scheduling problems. A few examples of such problems can be found in $[8,10,27]$ and the references therein. The main advantage of using the MLD formalism to model the MG components is that the final optimal scheduling problem turns out to be an MILP or MIQP problem $[8,10,27]$. Numerous commercial solvers are available to efficiently solve MILP and MIQP problems. HYSDEL (Hybrid System Description Language) permits application engineers to formulate hybrid system models in a descriptive fashion [28]. A compiler generates the MLD models from the descriptive HYSDEL models. The MLD models are subsequently used in optimization problems or for synthesizing controllers. In this paper, MLD models of all the DGs, BESSs, and ILs are obtained using HYSDEL. In the MLD framework, the overall system model is described using the following equations [29]:

$$
\begin{aligned}
& x(k+1)=A x(k)+B_{\mathrm{u}} u(k)+B_{\mathrm{aux}} w(k)+B_{\mathrm{aff}} \\
& E_{\mathrm{x}} x(k)+E_{\mathrm{u}} u(k)+E_{\mathrm{aux}} w(k) \leq E_{\mathrm{aff}},
\end{aligned}
$$

where $x=\left[\begin{array}{ll}x_{\mathrm{c}} & x_{\mathrm{b}}\end{array}\right]^{\mathrm{T}}, x_{\mathrm{c}} \in \mathbb{R}^{n_{\mathrm{x}}^{\mathrm{c}}}, x_{\mathrm{b}} \in\{0,1\}^{n_{\mathrm{x}}^{\mathrm{b}}}$, represents continuous and binary system states; $u=\left[\begin{array}{ll}u_{\mathrm{c}} & u_{\mathrm{b}}\end{array}\right]^{\mathrm{T}}, u_{\mathrm{c}} \in$ $\mathbb{R}^{n_{\mathrm{u}}^{\mathrm{c}}}, u_{\mathrm{b}} \in\{0,1\}^{n_{\mathrm{u}}^{\mathrm{b}}}$, represents continuous and binary system inputs; $w=\left[\begin{array}{ll}w_{\mathrm{c}} & w_{\mathrm{b}}\end{array}\right]^{\mathrm{T}}, w_{\mathrm{c}} \in \mathbb{R}^{n_{\mathrm{w}}^{\mathrm{c}}}, w_{\mathrm{b}} \in\{0,1\}^{n_{\mathrm{w}}^{\mathrm{b}}}$, represents continuous and binary auxiliary variables. Auxiliary variables are used in the MLD framework to convert propositional logic to linear inequalities of the form shown in (19). $A$, $B_{\mathrm{u}}, B_{\text {aux }}, B_{\text {aff }}, E_{\mathrm{x}}, E_{\mathrm{u}}, E_{\mathrm{aux}}$, and $E_{\text {aff }}$ are constant matrices of suitable dimensions which are used to describe the interactions between the system states, system inputs, and auxiliary variables. The auxiliary variables are solved with (19) using the current state of the system $x(k)$ and input $u(k)$, thereby determining the time evolution of the model described in (18) and (19). A well posed MLD system has a unique solution for the auxiliary variables $w(k)$ from (19) for a given state $x(k)$ and input $u(k)$. Interested readers may refer to [29] for a detailed description of the MLD framework. A detailed description of the modelling of thermal units and BESSs using the MLD approach can be found in [8] and [27], respectively.

\section{Energy Management System Architecture}

The proposed EMS comprises two sequential stages for minimizing the total MG operating cost. The motivation behind adopting this two-stage approach is to decrease the complexity of the overall optimization problem, thereby ensuring that it is solved within a reasonable time. The two stages of the proposed EMS are described below.

3.1. Stage 1: Unit Commitment. The UC problem for the MG is solved in Stage 1 wherein all the DGs, BESSs, pumps, and ILs are optimally scheduled to satisfy the active power demand. The overall optimization problem solved at this stage is described below.

$$
\min _{u, x, w} J=C_{\mathrm{DG}}+C_{\mathrm{BESS}}+C_{\mathrm{UCC}}+C_{\mathrm{Grid}}+C_{\mathrm{IL}}
$$

subject to (18), (19)

$$
\begin{aligned}
& u_{\min } \leq u \leq u_{\max } \\
& x_{\min } \leq x \leq x_{\max } \\
& w_{\min } \leq w \leq w_{\max }
\end{aligned}
$$




$$
\begin{aligned}
& P_{\mathrm{D}, k}-\sum_{h \in \mathscr{H}} P_{\mathrm{IL}, k}^{h}+P_{\mathrm{e}, k}^{\mathrm{loss}}+\sum_{m \in \mathscr{M}} P_{\mathrm{Pump}, k}^{m} \\
& =\sum_{f \in F} P_{\mathrm{DG}, k}^{f}+P_{\mathrm{eb}, k}-P_{\mathrm{es}, k} \\
& \quad+\sum_{e \in \mathscr{G}}\left(P_{\mathrm{bd}, k}^{e}-P_{\mathrm{bc}, k}^{e}\right)+\sum_{z \in \mathscr{\mathscr { T }}} P_{\mathrm{RES}, k}^{z},
\end{aligned}
$$

$\forall k \in \mathscr{K}$,

where the first constraint refers to the bounds on the system states, system inputs, and auxiliary variables. Equation (20) is the power balance constraint for the UC problem. The unexplained terms of (20) are described in the following paragraphs.

$C_{\text {Grid }}$ calculates the cost incurred by the MG due to the purchase of electricity from the utility grid. $C_{\text {Grid }}$ also accounts for the revenue earned from the sale of electricity to the utility grid. $C_{\text {Grid }}$ is calculated as follows:

$$
C_{\mathrm{Grid}}=\sum_{k \in K}\left(C_{\mathrm{p}, k} P_{\mathrm{eb}, k}-C_{\mathrm{s}, k} P_{\mathrm{es}, k}\right) .
$$

$C_{\mathrm{UCC}}$ is the cost incurred by the MG due to the import of uncontracted capacity from the utility grid. The uncontracted capacity is calculated based on the maximum demand as follows [25]:

$$
P_{\mathrm{UC}}=\max \left\{0, \max _{1 \leq k \leq 24}\left\{P_{\mathrm{eb}, k}-P_{\mathrm{CC}}\right\}\right\} .
$$

Equation (22) is linearized as follows:

$$
\begin{aligned}
P_{\mathrm{UC}} & \geq P_{\mathrm{eb}, k}-P_{\mathrm{CC}}, \quad \forall k \in \mathscr{K} \\
P_{\mathrm{UC}} & \geq 0, \\
C_{\mathrm{UCC}} & =U_{\mathrm{CC}} P_{\mathrm{UC}},
\end{aligned}
$$

where $U_{\mathrm{CC}}=\$ 12,860 / \mathrm{MW} /$ month and $P_{\mathrm{CC}}=0.7 \mathrm{MW}$. Due to its exorbitant pricing, uncontracted capacity needs to be avoided as much as possible. Efficient load management strategies can aid the EMS in achieving this target.

The overall optimization problem is solved in an MPC framework with a prediction horizon of 24. In Stage 1, the overall optimization problem turns out to be an MIQP problem. The optimization problem is described in MATLAB using YALMIP [30] and solved using CPLEX.

\subsection{Stage 2: Optimal Power Flow. Optimal power flow (OPF)} is a key optimization problem in power system operations. It is used to determine optimal setpoints for the system variables while satisfying power demand and respecting generator and network constraints. The main objective of the OPF problem is usually to minimize the power generation cost of the system. Importantly, the OPF problem also accounts for power losses in the system. Thus, the OPF problem can also be formulated to minimize power losses in the system. As the system load demand varies with time, it is essential to solve the OPF problem within a reasonable time. However, OPF is a nonconvex, nonlinear optimization problem which is NP-hard to solve [16]. Global optimization procedures to solve the OPF problem are computationally expensive. Notwithstanding their suboptimal results, gradient based methods have been widely adopted by researchers to solve the OPF problem due to their fast computational speeds. Among these, the quadratic programming method [31] and the interior point method (and its variants) [15, 32, 33] are popular due to their fast computational speeds.

3.2.1. Network Model. Let $\mathcal{N}$ and $\mathscr{L}$ represent the sets of $N$ buses and $L$ lines in the MG respectively. The generators are connected to a subset of $\mathcal{N}$. In this paper, multiple OPF problems are solved during different hours. Thus, all the variables and parameters of the OPF problem during hour $k$ are denoted by $(\cdot)_{k}$. In this formulation, the polar form of the complex bus voltage $v_{k} \in \mathbb{C}^{N}$ is used as $v_{k}^{i}=V_{k}^{i} e^{j \delta_{k}^{i}}$, where $V_{k}^{i}$ and $\delta_{k}^{i}$ are the magnitude and phase angle, respectively, of the voltage phasor $v_{k}^{i}$ at bus $i \in \mathcal{N}$. The complex power injection vector is denoted by $s_{k} \in \mathbb{C}^{N}$ such that $s_{k}^{i}=P_{\mathrm{e}, k}^{i}+j Q_{\mathrm{e}, k}^{i}$ for bus $i \in \mathcal{N}$, where $P_{\mathrm{e}, k}^{i}$ is the generated real power and $Q_{e, k}^{i}$ is the generated reactive power. All the transmission lines in the MG are modelled using the standard $\pi$-model. For transmission line $l$ connecting buses $i$ and $j ; l=(i, j) \epsilon$ $\mathscr{L}$, let $Y \in \mathbb{C}^{L}$ be the branch admittance matrix having components $Y_{i j}=g_{i j}+j b_{i j}$, where $g_{i j}$ and $b_{i j}$ are the series conductance and susceptance, respectively, and $b_{i j}^{\text {sh }}$ is the line charging susceptance. Also $d_{k} \in \mathbb{C}^{N}$ such that $d_{k}^{i}=P_{\mathrm{d}, k}^{i}+\mathrm{j} Q_{\mathrm{d}, k}^{i}$ for bus $i$, where $P_{\mathrm{d}, k}^{i}$ and $Q_{\mathrm{d}, k}^{i}$ are the active and reactive power demands at bus $i$, respectively, such that $P_{\mathrm{D}, k}=\sum_{i \in \mathcal{N}} P_{\mathrm{d}, k}^{i}$ and $Q_{\mathrm{D}, k}=\sum_{i \in \mathcal{N}} Q_{\mathrm{d}, k}^{i}$.

3.2.2. OPF Problem Formulation. The constraints for the OPF problem mainly adhere to Kirchhoff's laws and are formulated to ensure that the active and reactive powers are balanced at each bus while satisfying generation capability margins and voltage bounds. The constraints for the OPF problem are enumerated below.

(1) Active power balance at bus $i$ is

$$
\begin{aligned}
& P_{\mathrm{e}, k}^{i j}=g_{i j}\left(V_{k}^{i}\right)^{2}-g_{i j} V_{k}^{i} V_{k}^{j} \cos \left(\delta_{k}^{i j}\right) \\
& +b_{i j} V_{k}^{i} V_{k}^{j} \sin \left(\delta_{k}^{i j}\right) ; \quad i, j \in \mathcal{N}, \forall l \in \mathscr{L} \\
& P_{\mathrm{e}, k}^{i}=\sum_{f \in \mathscr{F}(i)} P_{\mathrm{DG}, k}^{f}+\sum_{e \in \mathscr{E}(i)} P_{\mathrm{BESS}, k}^{e}+P_{\mathrm{Grid}, k} \\
& +\sum_{z \in \mathscr{I}(i)} P_{\mathrm{RES}, k}^{z}-P_{\mathrm{d}, k}^{i}+\sum_{h \in \mathscr{H}(i)} P_{\mathrm{IL}, k}^{h} \\
& -\sum_{m \in \mathscr{M}(i)} P_{\mathrm{Pump}, k}^{m} \\
& P_{\mathrm{e}, k}^{i}=\sum_{j \in \mathscr{N}(i)} P_{\mathrm{e}, k}^{i j} ; \quad \forall i \in \mathcal{N}
\end{aligned}
$$




$$
\begin{aligned}
& P_{\mathrm{BESS}, k}^{e}=P_{\mathrm{bd}, k}^{e}-P_{\mathrm{bc}, k}^{e} ; \quad \forall e \in \mathscr{E} \\
& P_{\mathrm{Grid}, k}=P_{\mathrm{eb}, k}-P_{\mathrm{es}, k} .
\end{aligned}
$$

(2) Reactive power balance at bus $i$ is

$$
\begin{aligned}
Q_{\mathrm{e}, k}^{i j}= & \left(b_{i j}+\frac{b_{i j}^{\mathrm{sh}}}{2}\right)\left(V_{k}^{i}\right)^{2}-b_{i j} V_{k}^{i} V_{k}^{j} \cos \left(\delta_{k}^{i j}\right) \\
& -g_{i j} V_{k}^{i} V_{k}^{j} \sin \left(\delta_{k}^{i j}\right) ; \\
Q_{\mathrm{e}, k}^{i}= & \sum_{f \in \mathscr{F}(i)} Q_{\mathrm{DG}, k}^{f}+\sum_{e \in \mathscr{E}(i)} \mathrm{Q}_{\mathrm{BESS}, k}^{e}+Q_{\mathrm{Grid}, k} \\
& +\sum_{z \in \mathscr{Z}(i)} \mathrm{Q}_{\mathrm{RES}, k}^{z}-Q_{\mathrm{d}, k}^{i}+\sum_{h \in \mathscr{H}(i)} \mathrm{Q}_{\mathrm{IL}, k}^{h} \\
& -\sum_{m \in \mathscr{M}(i)} \mathrm{Q}_{\mathrm{Pump}, k}^{m} \\
Q_{\mathrm{e}, k}^{i}= & \sum_{j \in \mathscr{N}(i)} Q_{\mathrm{e}, k}^{i j} ; \quad \forall i \in \mathscr{N} \\
Q_{\mathrm{BESS}, k}^{e}= & Q_{\mathrm{bd}, k}^{e}-Q_{\mathrm{bc}, k}^{e} ; \quad \forall e \in \mathscr{E} \\
Q_{\mathrm{Grid}, k}= & Q_{\mathrm{eb}, k}-Q_{\mathrm{es}, k},
\end{aligned}
$$

where the active power flow $\left(P_{\mathrm{e}, k}^{i j}\right)$ and reactive power flow $\left(Q_{e, k}^{i j}\right)$ through line $l$ connecting buses $i$ and $j$ can be represented in (24a) and (25a) satisfying the physical laws of the power flow and $\delta_{k}^{i j}=\delta_{k}^{i}-\delta_{k}^{j} . P_{\mathrm{e}, k}^{i}$ and $Q_{\mathrm{e}, k}^{i}$ are the amounts of active and reactive power injections (if positive) or extractions (if negative) at bus $i$, respectively. It may be noted that all the reactive power variables (denoted using Q) follow a similar notation to the real power variables (denoted using $P$ ).

It is assumed that the pump loads and ILs consume reactive power equivalent to $50 \%$ of their active power consumption and that the converters of the BESSs and RESs at the PCC are capable of maintaining a power factor greater than 0.7. The sets of DGs, BESSs, RESs, ILs, and pumps connected to bus $i$ are denoted by $\mathscr{F}(i), \mathscr{E}(i), \mathscr{Z}(i), \mathscr{H}(i)$, and $\mathscr{M}(i)$, respectively; the set of buses connected to bus $i$ with transmission lines are denoted by $\mathscr{N}(i)$, where $\mathscr{F}(i) \subset$ $\mathscr{F}, \mathscr{E}(i) \subset \mathscr{E}, \mathscr{Z}(i) \subset \mathscr{Z}, \mathscr{H}(i) \subset \mathscr{H}, \mathscr{M}(i) \subset \mathscr{M}$, and $\mathscr{N}(i) \subset$ $\mathcal{N}$. Constraints (24c) and (25c) represent the active and reactive power balances at each bus, respectively. However, the additional variables $P_{\mathrm{e}, k}^{i j}, Q_{\mathrm{e}, k}^{i j}, P_{\mathrm{e}, k}^{i}$, and $Q_{\mathrm{e}, k}^{i}$ are omitted in the implementation.

(3) Active and reactive power injection limits of each power source are discussed in Section 3.3.

(4) Voltage bounds at bus $i$ are

$$
V_{k}^{i} \in\left[V_{\min }^{i}, V_{\max }^{i}\right] ; \quad \forall i \in \mathcal{N}
$$

where $(\cdot)_{\min }$ and $(\cdot)_{\max }$ indicate the lower and upper bounds of the corresponding decision variable, respectively.
3.3. Coordination between Stage 1 and Stage 2. The EMS solves Stage 1 and Stage 2 alternately. In Stage 1, binary statuses and dispatch values for all the DGs, BESSs, pumps, and ILs in the MG are determined. In addition to this, a schedule for exchanging power with the utility grid is also determined. The power exchange schedule and dispatch values of the DGs, BESS, pumps, and ILs are shared with the OPF problem in Stage 2 . The OPF problem is formulated by permitting a small degree of freedom around the scheduled power exchange values and dispatch values of the DGs and BESSs generated in Stage 1. In Stage 2, the network power losses are determined and power flow convergence is checked. The power losses are shared with Stage 1 which solves the UC problem including the power losses. The dispatch and power exchange values are shared with Stage 2. This iterative process continues till convergence. This process is illustrated in the flowchart shown in Figure 1. The first UC problem in Stage 1 is solved with an initial $\left(P_{\mathrm{e}, k}^{\text {loss }}\right)=0 \mathrm{MW}$ for each hour. The status and dispatch vectors are then passed to Stage 2. Based on these values, the bounds of the controllable generator dispatch variables in the OPF subproblems are modified as shown below. Here, the DGs, BESSs, and the main grid supply are considered as controllable generators. Let $\widehat{P}_{\mathrm{e}, k}^{g}, \widehat{u}_{k}^{g}$, and $\widehat{P}_{\mathrm{BESS}, k}^{e}$ be the dispatch value, the commitment status for generator $g$, and the power flow from BESS $e$ during hour $k$, respectively, received from Stage 1.

$$
\begin{aligned}
& \text { For } f^{\text {th }} \mathrm{DG}, \\
& \begin{array}{r}
P_{\mathrm{e}, k}^{f} \geq \widehat{u}_{k}^{f} \max \left\{(1-\alpha) \widehat{P}_{\mathrm{e}, k}^{f}, P_{\mathrm{e}, \min }^{f}\right\}, \\
\forall k \in K, \forall f \in \mathscr{F} \\
P_{\mathrm{e}, k}^{f} \leq \widehat{u}_{k}^{f} \min \left\{(1+\alpha) \widehat{P}_{\mathrm{e}, k}^{f}, P_{\mathrm{e}, \max }^{f}\right\}, \\
\forall k \in K, \forall f \in \mathscr{F} \\
\widehat{u}_{k}^{f} Q_{\mathrm{e}, \min }^{f} \leq Q_{\mathrm{e}, k}^{f} \leq \widehat{u}_{k}^{f} Q_{\mathrm{e}, \max }^{f}, \quad \forall k \in K, \forall f \in \mathscr{F} .
\end{array}
\end{aligned}
$$

The power supplies from the grid and the BESSs are bidirectional. Therefore, based on the power flow direction, the shifted domain is defined as follows.

$$
\begin{aligned}
& \text { For } e^{\text {th }} \mathrm{BESS} \text {, } \\
& \qquad \mathrm{Q}_{\mathrm{BESS}, \mathrm{min}, k}^{e} \leq \mathrm{Q}_{\mathrm{BESS}, k}^{e} \leq \mathrm{Q}_{\mathrm{BESS}, \max , k}^{e} ; \quad \forall e \in \mathscr{E} .
\end{aligned}
$$

(1) If $\widehat{P}_{\mathrm{bd}, k}^{e} \geq 0\left(\widehat{P}_{\mathrm{BESS}, k} \geq 0\right)$,

$$
\begin{aligned}
& P_{\mathrm{BESS}, k}^{e} \geq \max \left\{(1-\alpha) \widehat{P}_{\mathrm{BESS}, k}^{e}, 0\right\} \\
& P_{\mathrm{BESS}, k}^{e} \leq \min \left\{(1+\alpha) \widehat{P}_{\mathrm{BESS}, k}^{e}, P_{\mathrm{bd}, \max }^{e}\right\} .
\end{aligned}
$$

(2) If $\widehat{P}_{\mathrm{bc}, k}^{e} \geq 0\left(\widehat{P}_{\mathrm{BESS}, k} \leq 0\right)$,

$$
\begin{aligned}
& P_{\mathrm{BESS}, k}^{e} \geq \min \left\{(1-\alpha) \widehat{P}_{\mathrm{BESS}, k}^{e}, 0\right\} \\
& P_{\mathrm{BESS}, k}^{e} \leq \max \left\{(1+\alpha) \widehat{P}_{\mathrm{BESS}, k}^{e},-P_{\mathrm{bc}, \max }^{e}\right\} .
\end{aligned}
$$

Note that the shifted domains for the grid supply also can be defined similar to (28), (29a), (29b), (30a), and (30b) based on 


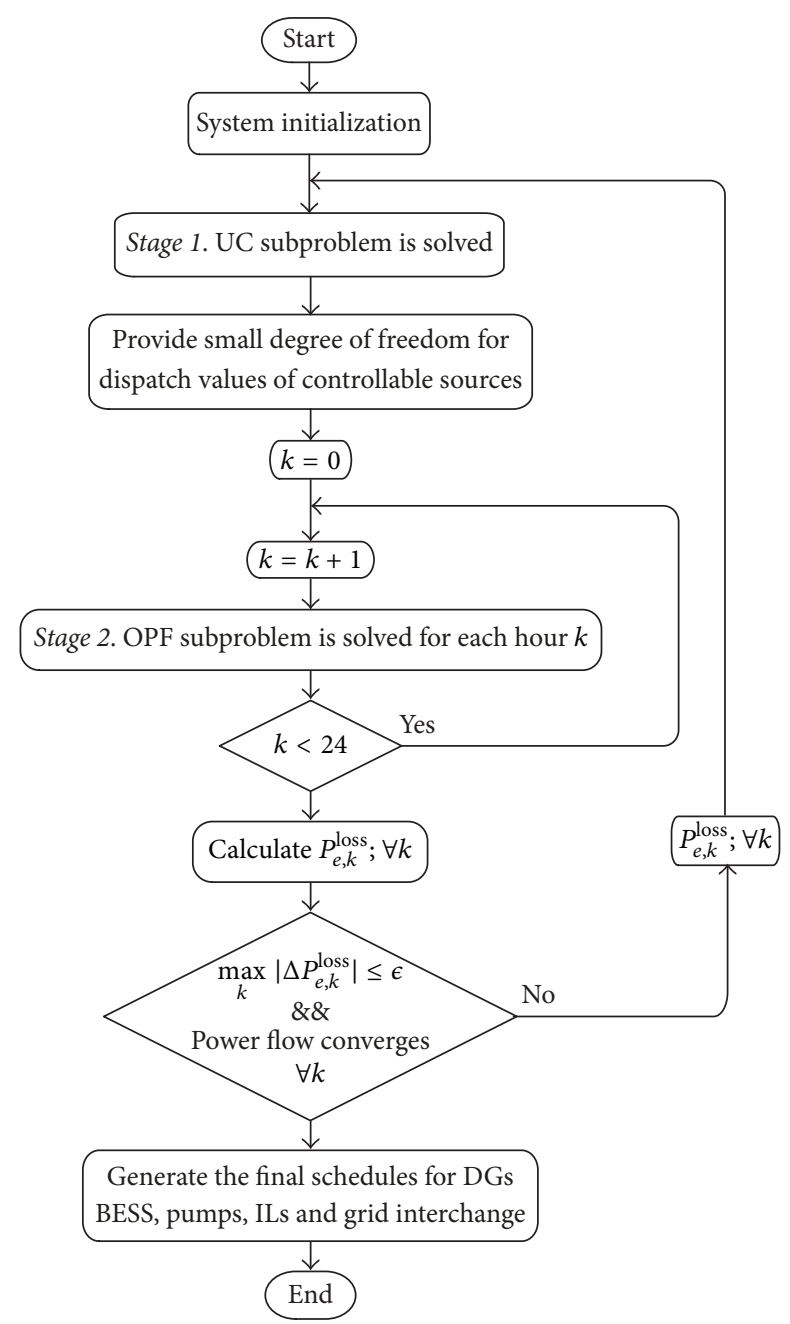

FIGURE 1: Flowchart for EMS layer computations.

the power flow direction. Here, $\alpha$ is a positive parameter value denoting the small degree of freedom permitted around the optimal dispatch values calculated in Stage 1. This parameter should be wisely chosen as a lower $\alpha$ leads to slower convergence and a higher $\alpha$ will result in the connection between Stage 1 and Stage 2 being lost. In this paper, $\alpha$ is taken as 0.03 . The uncontrollable sources such as the RESs are fixed in a similar manner to Stage 1.

Importantly, the OPF problems solved in Stage 2 are decoupled from each other. Hence, parallel solving techniques can be used to further increase the computational performance if necessary. The OPF problem in Stage 2 computes the power loss during each hour. This calculation is shown below in (31a). The total power loss $\left(P_{e, k}^{\text {loss }}\right)$ during each hour $k$ is conveyed to the UC problem in Stage 1 for the next iteration. Thereafter, the UC problem is solved once again in Stage 1 with losses included and the dispatch values are shared with Stage 2.

$$
\begin{aligned}
P_{\mathrm{e}, k}^{\text {loss }}=\sum_{l \in \mathscr{L}}\left[P_{\mathrm{e}, k}^{i j}+P_{\mathrm{e}, k}^{j i}\right] & \\
& \quad i, j \in \mathscr{N}, \forall l=(i, j) \in \mathscr{L}, \forall k \in \mathscr{K} .
\end{aligned}
$$

The algorithm progresses iteratively till the power losses and the dispatch values converge. The proof of convergence for this method was demonstrated in [15].

\section{Case Studies}

Two exemplar MGs are modelled in this paper. First, the optimal scheduling of a modified IEEE 30-bus system is performed under the following operational scenarios to demonstrate the efficacy of the proposed EMS and pump scheduling formulations:

(1) Without optimal pump scheduling and ILs, water is pumped in the shortest possible time using only the 3 main pumps.

(2) Optimal pump scheduling is performed using the 3 main pumps alone.

(3) Optimal pump scheduling is performed using only the 3 main pumps and the 4 auxiliary pumps.

(4) Optimal pump scheduling is performed using only the 3 main pumps and the 3 ILs.

(5) Optimal pump scheduling is performed using the 3 main pumps, the 4 auxiliary pumps, and the 3 ILs.

Subsequently, to further validate the versatility and efficacy of the proposed EMS for different networks, the optimal scheduling of a modified IEEE 57-bus system is performed under Scenario 5.

4.1. Case Study 1: Optimal Scheduling of a Modified IEEE 30-Bus System. A modified IEEE 30-bus system is adopted as an exemplar MG for this case study. The base value is considered as $8000 \mathrm{kVA}$ and the line resistance and reactance values are increased to 3 and 1.5 times the p.u. values provided in the standard MATPOWER case file for the IEEE 30-bus system, respectively [34]. In the 30-bus system used in this paper, the three DGs are connected to buses 27, 2, and 3, respectively, while the BESS and wind farm are connected to bus 22. The solar PV plant is connected to bus 13 while bus 1 serves as the point of common coupling (PCC) with the main utility grid. The 3 main pumps are connected to bus 27 while the 4 auxiliary pumps are connected to bus 29 . Finally, the summation of ILs is distributed among the load buses in proportion to their nominal load demand.

4.1.1. System Initialization. For all the simulation scenarios, it was assumed that the main pumps were switched off prior to the start of the optimization period. For Scenarios 3 and 5 , it was assumed that the auxiliary pumps were switched off prior to the start of the optimization period. Furthermore, it was assumed that $\mathrm{SOC}_{1}^{e}=0.6$. Finally, it was assumed that all the DGs in the MG were switched off prior to the start of the optimization period.

All the scenarios were simulated under the assumption that accurate point forecasts for load demand (excluding pump loads), electricity prices, and RES generation were available. The electricity price forecasts in this paper were adapted from $[35,36]$. The forecasts for load demand (excluding pump loads), RES generation, and electricity prices in 

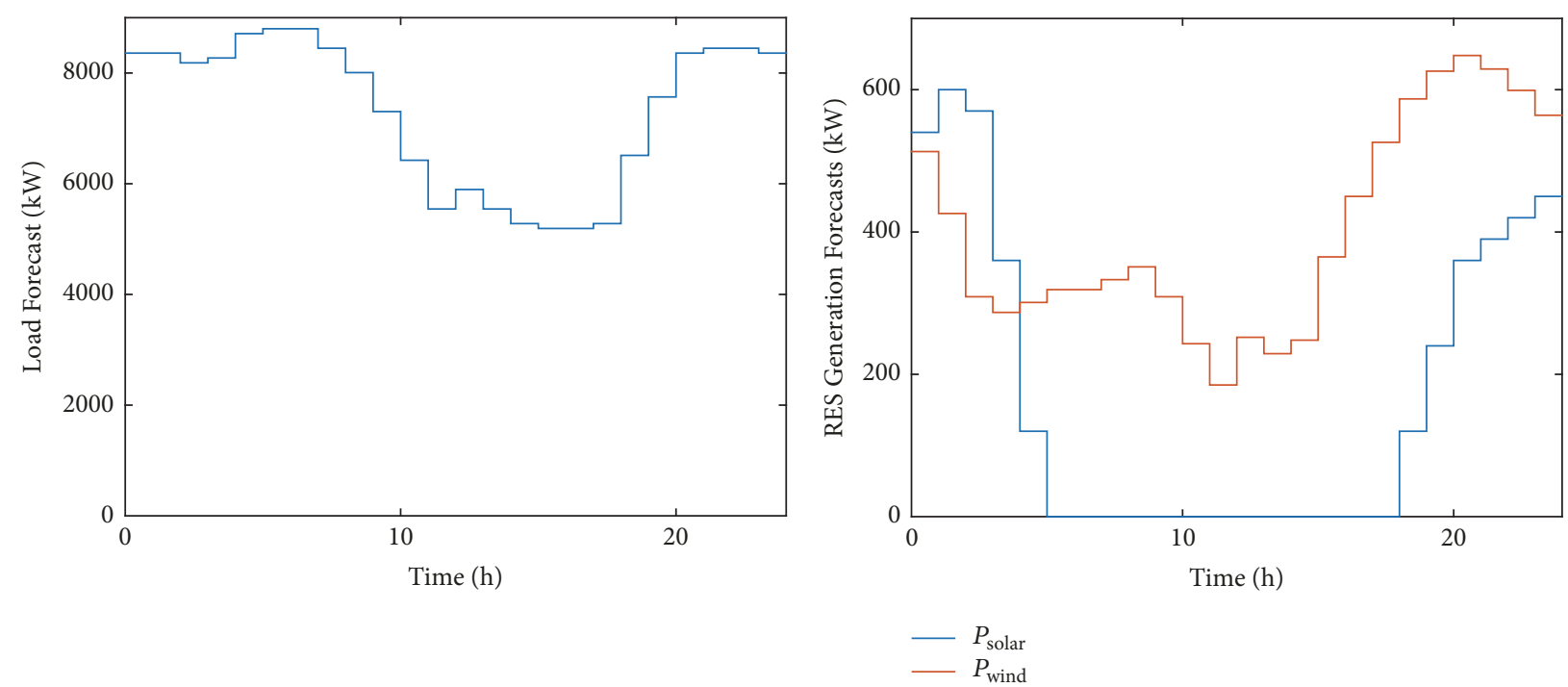

(a)

(b)

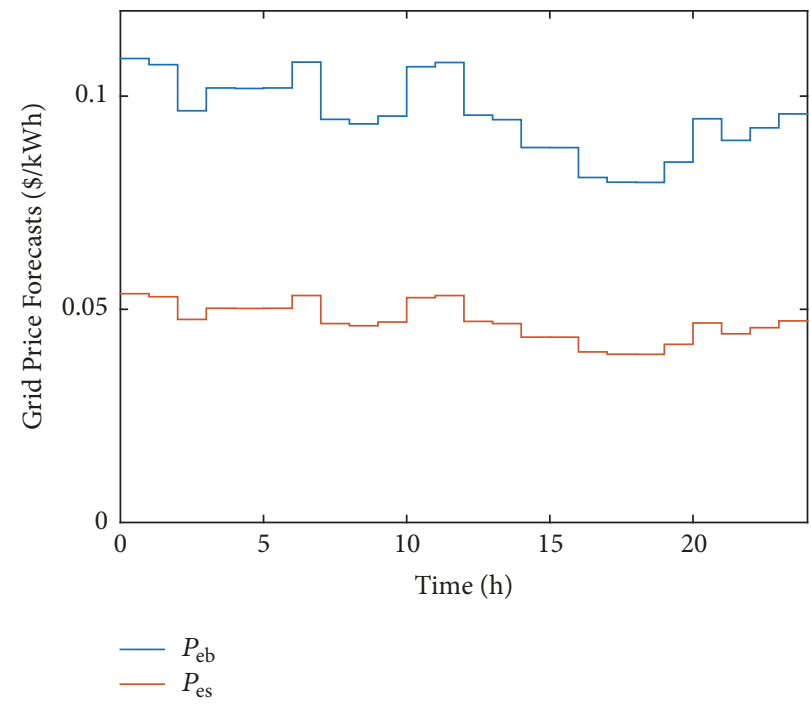

(c)

FIGURE 2: Forecasts of (a) load demand, (b) RES generation, and (c) electricity price.

this case study are shown in Figures 2(a), 2(b), and 2(c), respectively.

4.1.2. Optimal Scheduling Results. The final dispatch values of the DGs in Scenarios 1-5 are shown in Figures 3(a), 4(a), 5(a), $6(\mathrm{a})$, and $7(\mathrm{a})$, respectively. The BESS charge and discharge profiles in Scenarios 1-5 are shown in Figures 3(b), 4(b), 5(b), $6(\mathrm{~b})$, and 7(b), respectively. The power purchased from the main grid, $P_{\mathrm{eb}}$, in Scenarios 1-5 is shown in Figures 3(c), 4(c), $5(\mathrm{c}), 6(\mathrm{c})$, and $7(\mathrm{c})$, respectively. The schedules for the main pumps in Scenarios 1-5 are shown in Table 3. The auxiliary pump schedules are provided in Table 4 . The IL curtailment schedules in Scenarios 4 and 5 have been illustrated in Figures 8 and 9 , respectively.

In Scenario 1, it is assumed that the 3 main pumps are switched on during the first 3 hours of the day in order to pump the water in the shortest possible time. This action has major cost implications since the overall system load demand is high during the first 3 hours of the day. This is evidenced by the results shown in Table 2. From Figure 3(c), it is observed that $P_{\mathrm{eb}}$ exceeds the contracted capacity of $0.7 \mathrm{MW}$ during the first 3 hours. As a result, an uncontracted capacity charge of $\$ 11,991.5$ is incurred. It is also observed from Figure 3(c) that the EMS purchases electricity from the utility grid beyond the contracted capacity even during hours when the total load demand is lesser. This can be attributed to the methodology used to calculate the uncontracted capacity cost shown in (22) and (23). Consequently, from Figure 3(a), it is seen that DG 3 is shutdown from hour 6 onwards and DG 2 is shutdown between hours 14 and 17. From hour 18 onwards, when load demand starts increasing, DG 2 is once again started up and operated at full capacity. The relatively cheaper DG 1 is operated at full capacity throughout the day. 


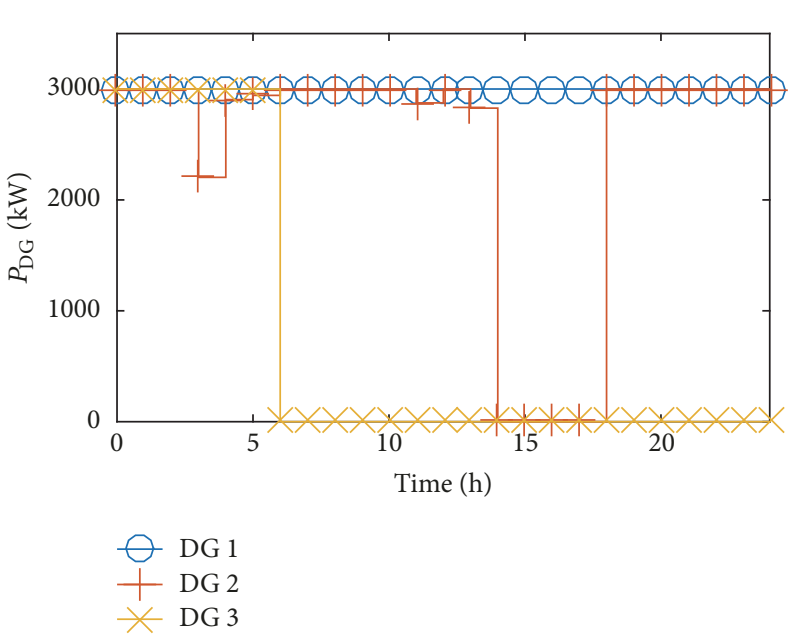

(a)

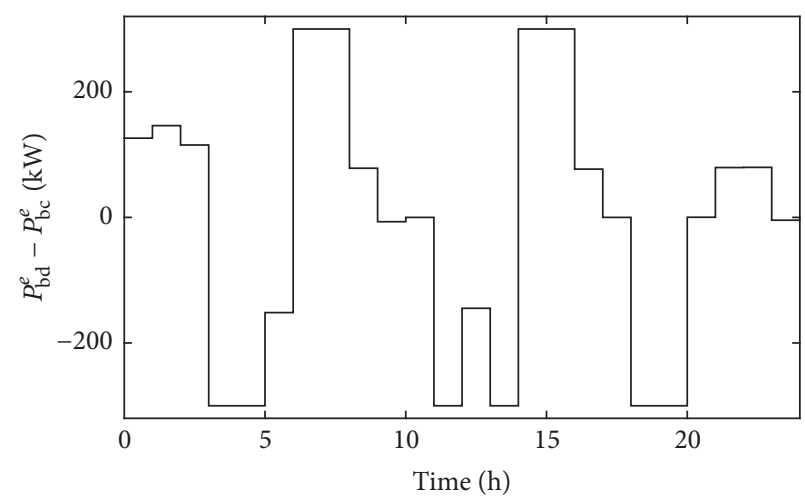

(b)

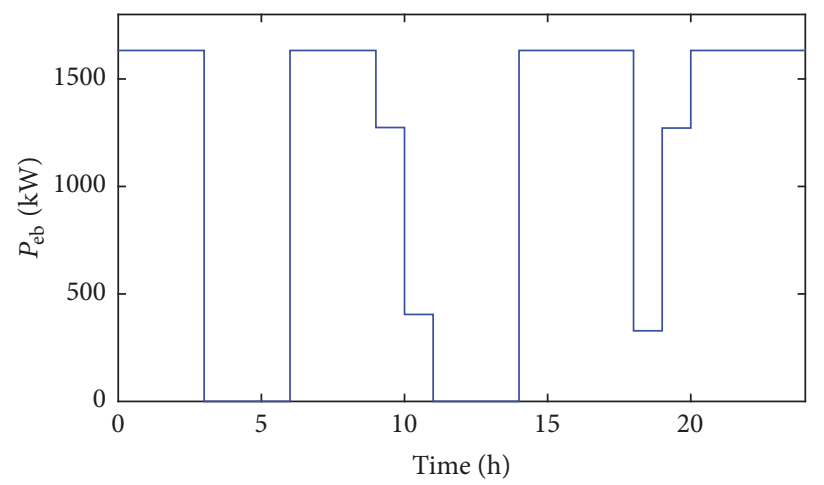

(c)

FIGURE 3: Scenario 1: (a) dispatch values of DG 1, DG 2, and DG 3, (b) BESS charge and discharge profiles, and (c) $P_{\mathrm{eb}}$.

TABLE 2: Cost breakdown and computational times for Scenarios 1-5.

\begin{tabular}{lccccc}
\hline $\begin{array}{l}\text { Scenario } \\
\#\end{array}$ & $\begin{array}{c}\text { Uncontracted } \\
\text { capacity cost } \\
(\$)\end{array}$ & $\begin{array}{c}\text { Interruptible } \\
\text { load cost }(\$)\end{array}$ & Total cost $(\$)$ & $\begin{array}{c}\text { Percentage } \\
\text { reduction in } \\
\text { total cost }\end{array}$ & $\begin{array}{c}\text { Computational } \\
\text { time }(\mathrm{s})\end{array}$ \\
\hline 1 & $11,991.50$ & - & $34,710.10$ & - & 38.11 \\
2 & 0 & - & $29,997.80$ & $13.58 \%$ & 60.59 \\
3 & 0 & - & $29,945.11$ & $13.73 \%$ & 136.04 \\
4 & 0 & 864.21 & $22,995.63$ & $33.75 \%$ & 60.81 \\
5 & 0 & 861.90 & $22,911.79$ & $33.99 \%$ & 108.53 \\
\hline
\end{tabular}

From Table 2, it is observed that the introduction of pump scheduling in Scenario 2 eliminates the uncontracted capacity charge incurred in Scenario 1. From Figure 4(a), it is observed that all 3 DGs are operated during the hours of peak load demand (hours 0-10 and 20-24). From Figure 4(c), it is clear that the maximum power imported from the main grid does not exceed 0.7 MW. From Figure 4(b), it is seen that the BESS usage in Scenario 2 is much lower than what is observed in Scenario 1. In Scenario 1, it was observed that the MG was forced to import uncontracted capacity during the first 3 hours of the day to meet the high load demand. Due to this, the EMS continued importing power from the main grid during the later hours of the day as well. Due to the elimination of the uncontracted capacity in Scenario 2, DG 2 is operated throughout the day. It is clear from Table 3 that the pump scheduling ensures that only 1 pump is operated at a time to avoid uncontracted capacity. Moreover, from Table 3, it is observed that main pumps 1 and 3 are operated during the first 4 hours and last 4 hours, respectively. During these hours, all 3 DGs are operational thereby ensuring that the MG has enough running capacity to accommodate the main pumps.

Compared to Scenario 2, a reduction of 1 hour in main pump usage is observed in Scenario 3 due to the introduction of auxiliary pumps. Consequently, as observed in Table 2, there is a marginal reduction of $\$ 53$ in the total cost for 


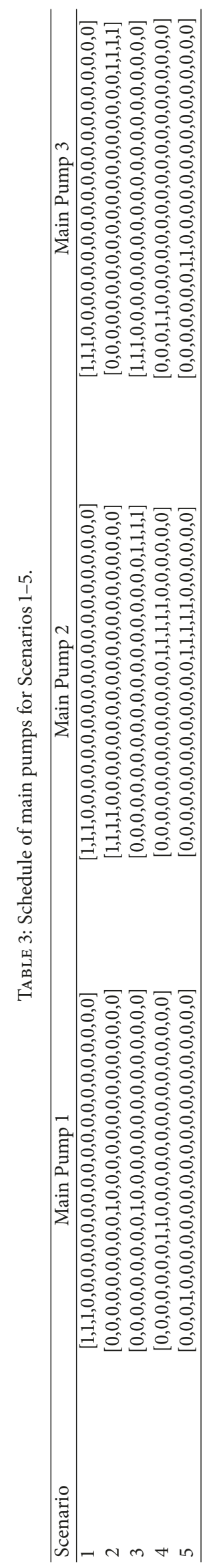




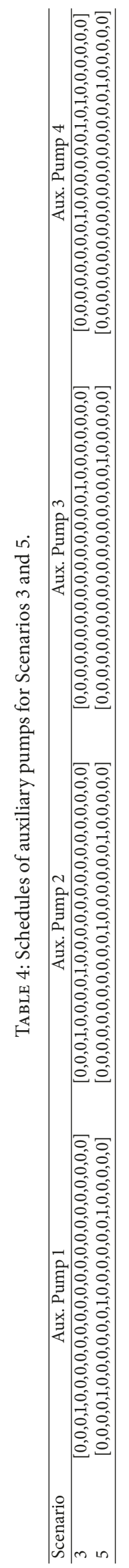



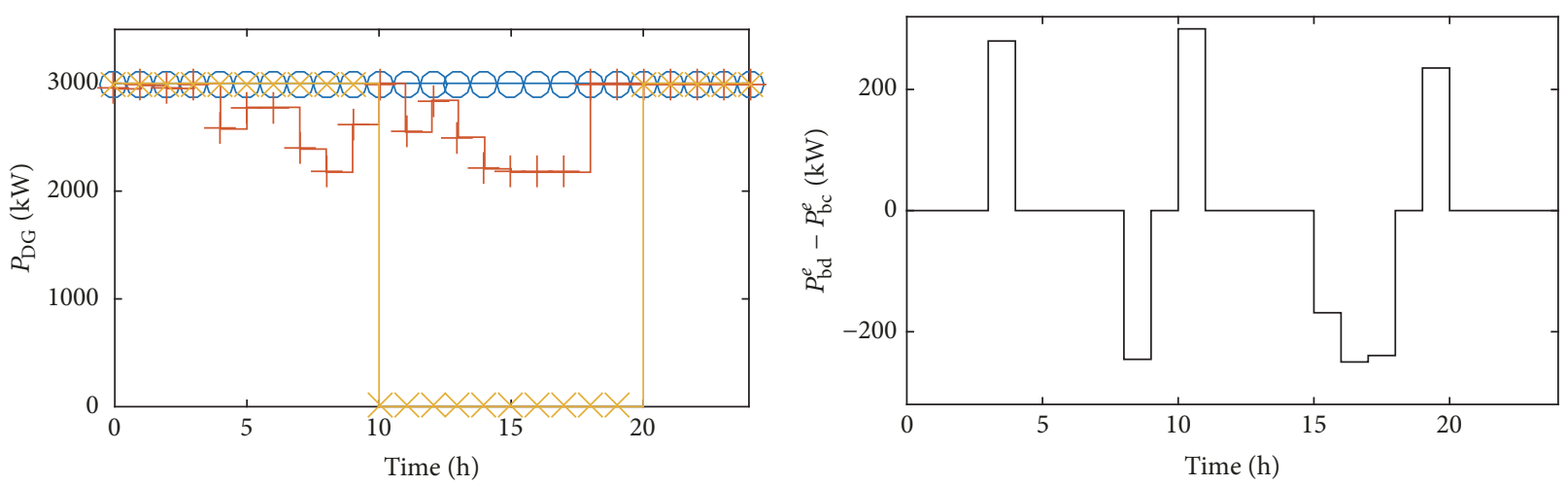

(a)

(b)

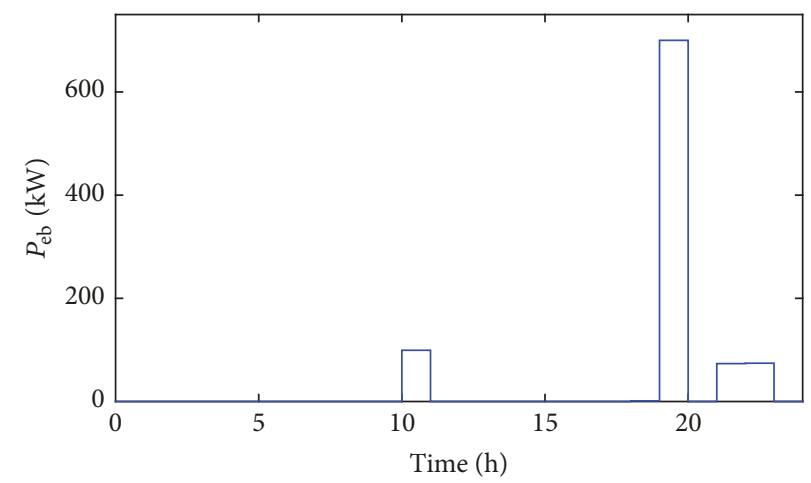

(c)

FIGURE 4: Scenario 2: (a) dispatch values of DG 1, DG 2, and DG 3, (b) BESS charge and discharge profiles, and (c) $P_{\mathrm{eb}}$.

Scenario 3 when compared with Scenario 2. The schedules of main pumps, dispatch of DGs, BESS charge and discharge profiles, and trends in electricity purchase from the main grid in Scenario 3 are similar to Scenario 2.

As observed from Table 2, the introduction of ILs in Scenario 4 leads to a reduction of $\$ 6,949$ in the total cost when compared with Scenario 3. From Figure 8, it is clear that the ILs are mainly utilized during the first 3 hours and the last 5 hours of the day when the load demand is quite high. Compared to the previous scenarios, the usage of the relatively expensive DG 3 is significantly reduced in Scenario 4. As observed from Figure 6(a), the EMS keeps the relatively expensive DG 3 switched off during the first 3 hours. However, DG 3 needs to be switched on at hour 4 since the total curtailment permitted for each IL in a day is capped at $2 \mathrm{MWh}$. As shown in Figure 6(c), the main grid electricity makes up for any shortfall without exceeding the contracted capacity as and when required. It is observed from Table 3 that the main pumps are mostly used during the valleys in the load profile.

The trends in Figures 7(a)-7(c) are quite similar to Figures 6(a)-6(c). As with Scenario 3, the inclusion of auxiliary pumps leads to a marginal decrease in the total cost of Scenario 5 when compared with Scenario 4 . However, from Table 2, it is seen that the total cost in Scenario 5 (lowest cost scenario) is $33.99 \%$ lower than the total cost in Scenario 1 which is the worst case. In Scenario 5, unlike Scenario 4, a main pump is not operated during hour 5. From Figures 7(b) and 9, it is seen that this eliminates the need to import power from the main grid and curtail ILs during hour 5 as was the case in Scenario 4.

Figure 10 illustrates the convergence of the 2-stage EMS computations described in Section 3. From Figure 10, it is observed that Scenario 3 takes more number of iterations to converge. From Figure 10, it is observed that the total operating costs of all the scenarios reach close to their final values after 3 iterations. It may be noted that the trajectories followed by both total operating cost and total power loss are similar. Finally, the sale of electricity to the utility grid was not observed in any of the scenarios considered.

4.2. Case Study 2: Optimal Scheduling of a Modified IEEE 57Bus System. A modified IEEE 57-bus system is adopted as an exemplar MG for this case study. The optimal scheduling of the modified 57-bus MG is performed under Scenario 5. The base value is considered as $7000 \mathrm{kVA}$ and the line reactance values are decreased by $50 \%$ when compared with the p.u. values provided in the standard MATPOWER case file for the IEEE 57-bus system [34]. In the 57-bus system used in this paper, the three DGs are connected to buses 1, 8, and 9, 


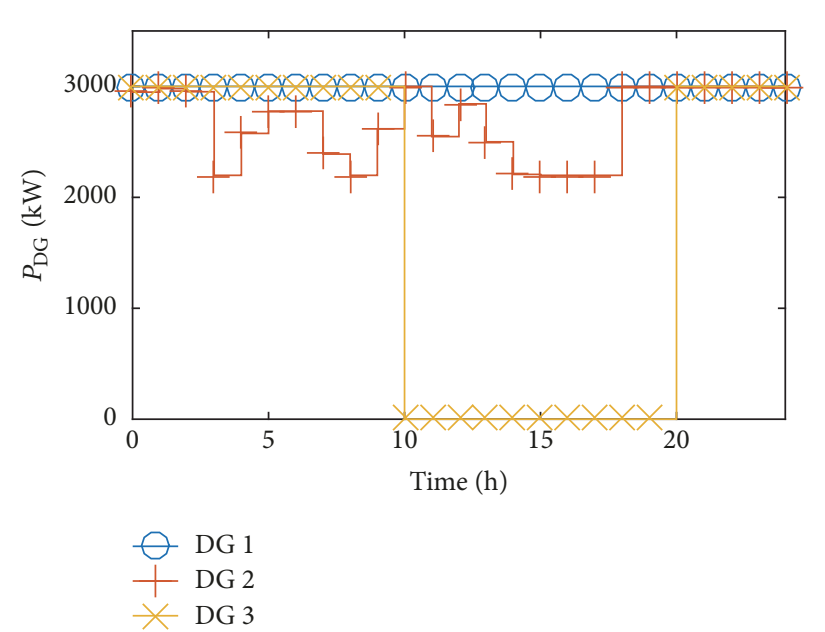

(a)

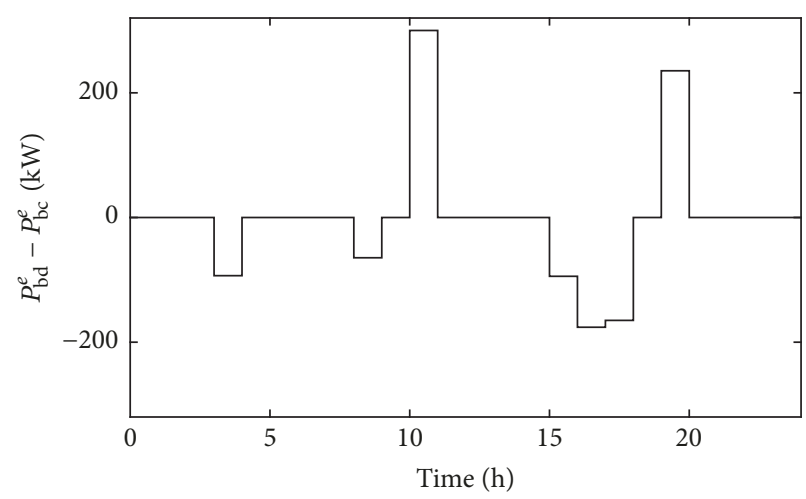

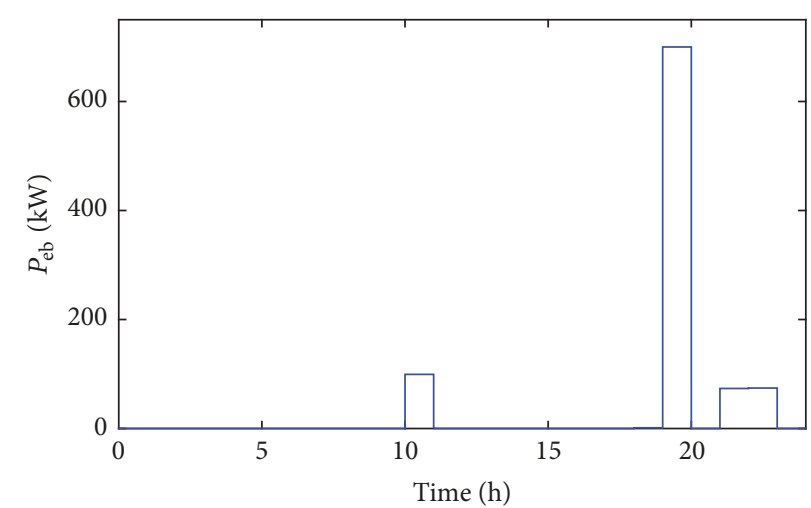

(b)

(c)

FIGURE 5: Scenario 3: (a) dispatch values of DG 1, DG 2, and DG 3, (b) BESS charge and discharge profiles, and (c) $P_{\mathrm{eb}}$.

respectively, while the wind farms are connected to buses 2 and 12. BESSs are connected to buses 2 and 12. A solar PV plant is connected to bus 3 while bus 6 serves as the point of common coupling (PCC) with the main utility grid. The 3 main pumps are connected to bus 2 while the 4 auxiliary pumps are connected to bus 1. Finally, the summation of ILs is distributed among the load buses in proportion to their nominal load demand.

4.2.1. System Initialization. For this case study, it was assumed that all the main pumps and auxiliary pumps were switched off prior to the start of the optimization period. Furthermore, it was assumed that initial SOCs for the two BESSs in the MG were 0.6 and 0.5 , respectively. Finally, it was assumed that all the DGs in the MG were switched off prior to the start of the optimization period.

This case study was performed under the assumption that accurate point forecasts for load demand (excluding pump loads), electricity prices, and RES generation were available. The load demand forecast for this case study is shown in Figure 11. Forecasts of the solar PV plant and one wind power plant in this case study are shown in Figure 2(b) while the forecast for the second wind power plant in this case study is also shown in Figure 11.
4.2.2. Optimal Scheduling Results. The final dispatch values of the DGs in this case study are shown in Figure 12(a). The charge and discharge profiles of the BESSs in this case study are shown in Figure 12(b). The power purchased from the main grid in this case study, $P_{\mathrm{eb}}$, is shown in Figure 12(c). In this case study, Main Pump 1 operates between hours 16 and 18; Main Pump 2 operates only during hour 19; Main Pump 3 operates between hours 12 and 15 . Auxiliary Pump 1 operates during hours 15, 17, and 18; Auxiliary Pump 2 operates during hours 11 and 15; Auxiliary Pump 3 operates during hour 15; and Auxiliary Pump 4 operates during hour 11.

The results of this case study largely agree with those of Case Study 1 . The pumps are generally operated during the valley periods in the load profile which coincide with the late night hours. A clear indication of this is the operation of 1 main pump and 3 auxiliary pumps during hour 15. From Figure 11, it is observed that the load demand is at its lowest during hour 15. The EMS avoids using the expensive DG 3 due to the higher contribution from the RESs in this case study. DGs 1 and 2 are used at nearly their full capacities throughout the 24-hour period. From Figure 13, it is observed that the ILs are utilized during the hours when the load demand is at its peak. During these hours, the EMS also imports power from the utility grid without exceeding the contracted capacity of 

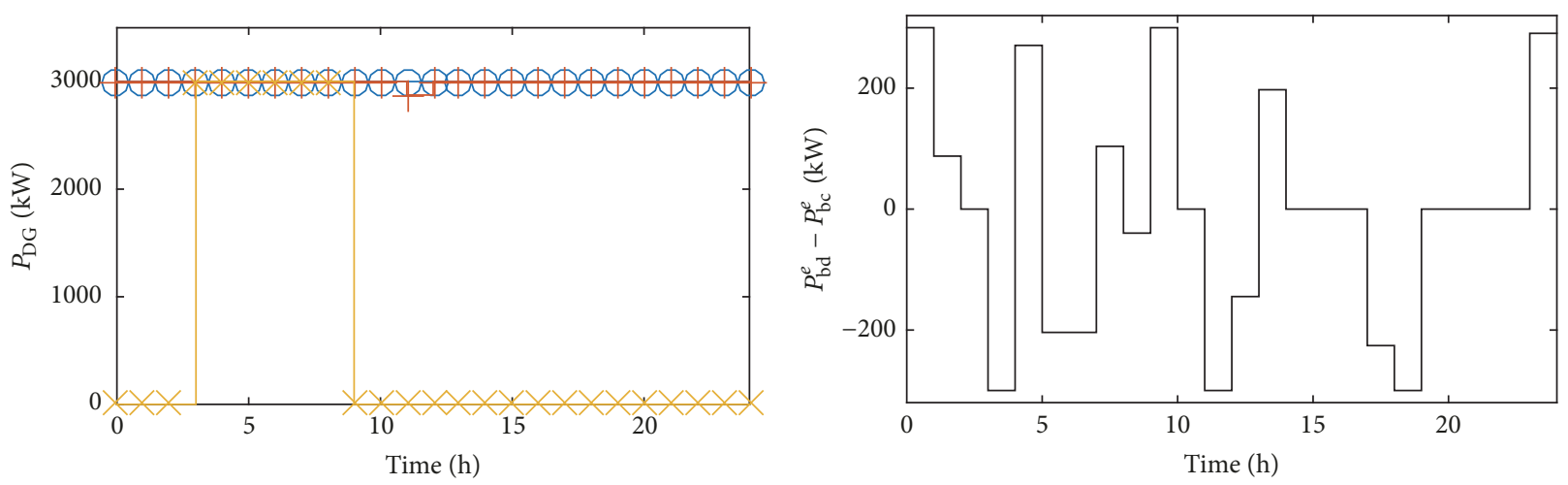

(a)

(b)

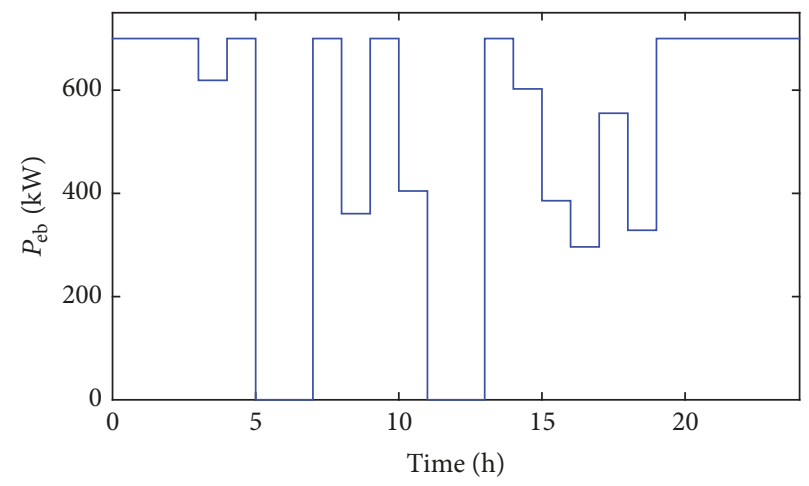

(c)

FIGURE 6: Scenario 4: (a) dispatch values of DG 1, DG 2, and DG 3, (b) BESS charge and discharge profiles, and (c) $P_{\mathrm{eb}}$.

0.8 MW. From Figure 12(b), it is observed that the BESSs discharge during some peak loading hours while charging during the valley periods in the load profile. The overall MG cost in this case study was $\$ 15842.79$ while the computational time taken to solve the problem was $154.49 \mathrm{~s}$. The higher contribution from the RESs results in a lower cost in this case study.

Finally, Figure 14 illustrates the convergence of the 2-stage EMS computations. From Figure 14, it is observed that the EMS takes more number of iterations to converge compared to all the scenarios in Case Study 1 except Scenario 3. Finally, the sale of electricity to the utility grid was not observed in this case study.

4.3. Discussions and Scope for Future Work. This section discusses the limitations of this paper and how the assumptions made could potentially impact the optimal MG scheduling results. Furthermore, some possible future research directions are also presented. Case Study 1 clearly established the potential of load management strategies in reducing the MG operating cost. In Scenario 1, it was assumed that the main pumps would be switched on during the first 3 hours of the day to pump out the water in the fastest possible time. Even if this assumption was not true, load management strategies such as pump scheduling and curtailment of ILs still provide the maximum reduction in operation cost without compromising on any operational requirements.

The MG models in this paper were developed using the MLD approach which essentially results in a state-space representation of the system as shown in (18). Consequently, the system states need to be initialized before starting the simulation. The initial system states provide a snapshot of the MG prior to the start of the simulation. The initialization of the system states needs to be done carefully to ensure that all the constraints are respected and the overall tractability of the problem is not affected. In line with these requirements, the authors had randomly selected a set of values to initialize the system states. However, it is clear that the selection of different initial states for the system would result in different system evolution according to (18) and (19) and consequently different scheduling results.

In this paper, the optimal MG scheduling is performed on the basis of fixed forecasts for RES generation, electricity price, and load demand. In case of any uncertainties in the RES generation and electricity price forecasts, the resulting MG schedules which are generated by the EMS may be suboptimal. One way to deal with the uncertainties is to introduce spinning reserve constraints in the scheduling problem which would enable the MG to manage the loss of the largest RES. The objective of this paper was to present 

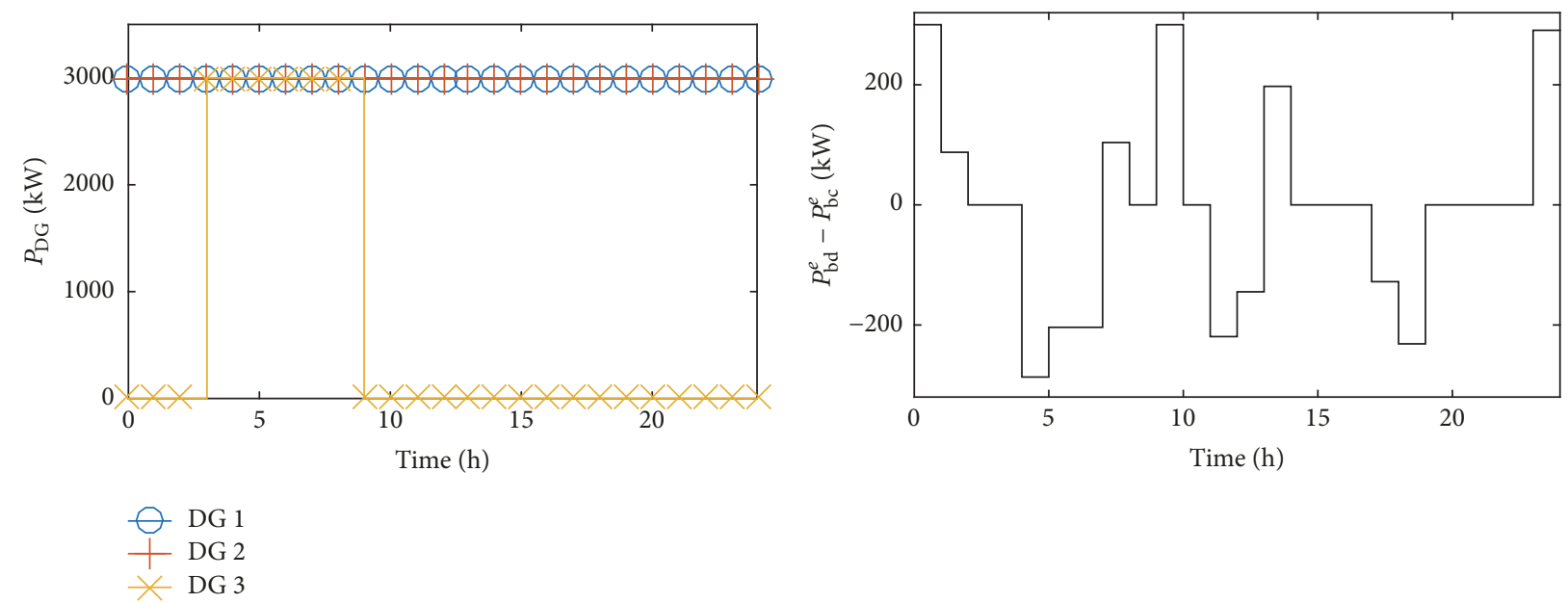

(a)

(b)

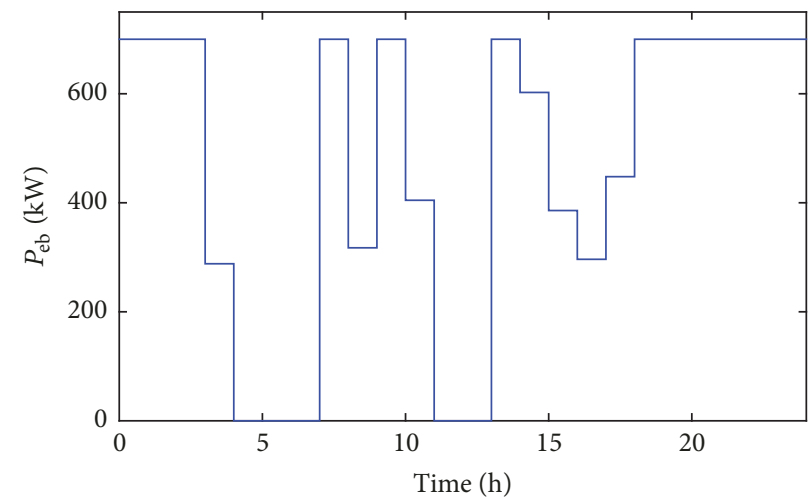

(c)

FIGURE 7: Scenario 5: (a) dispatch values of DG 1, DG 2, and DG 3, (b) BESS charge and discharge profiles, and (c) $P_{\mathrm{eb}}$.

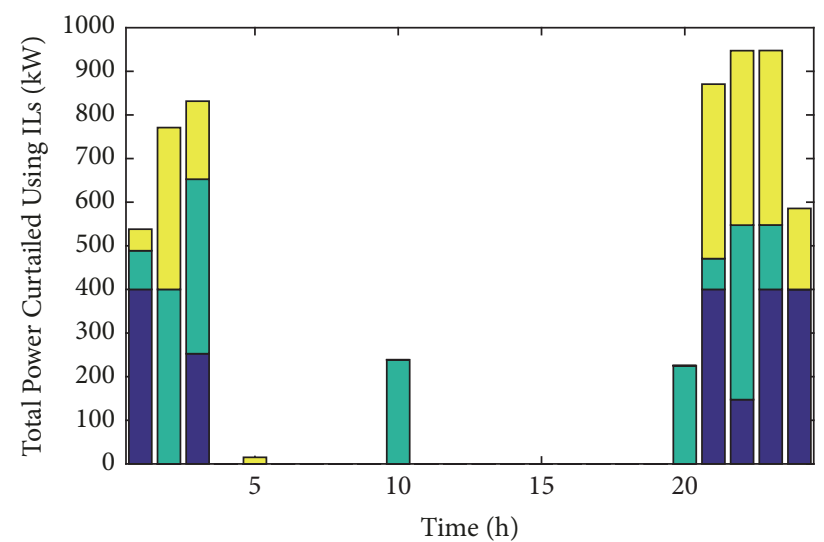

$\square$ IL 2

IL 3

FIGURE 8: Curtailment of ILs in Scenario 4.

the EMS architecture and problem formulation. The authors have not focused on handling uncertainties in this work. In this context, one possible direction for future work is to
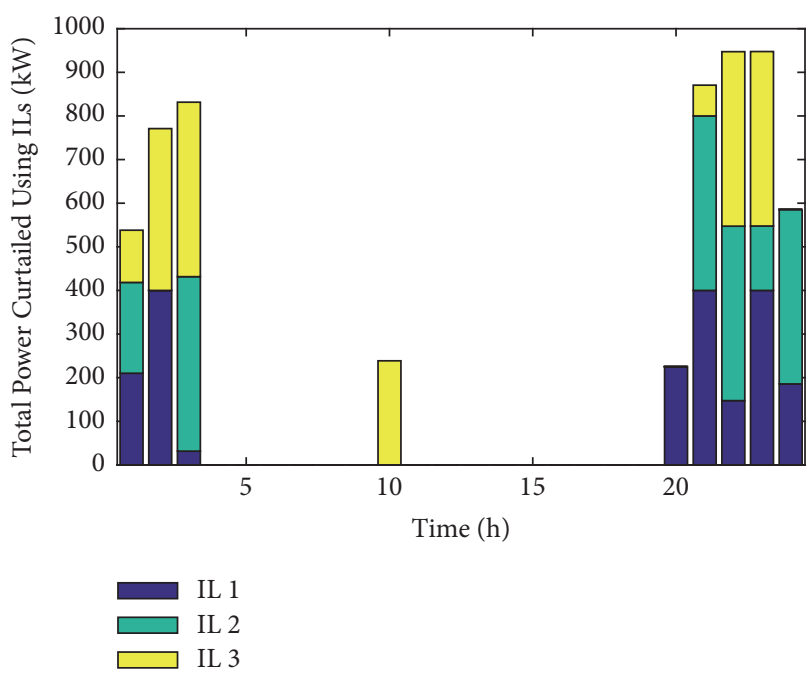

FIGURE 9: Curtailment of ILs in Scenario 5.

develop a scenario-based robust optimization approach for handling forecast uncertainties in the optimal scheduling of MGs while also respecting the network constraints using the EMS architecture explained in this paper. 


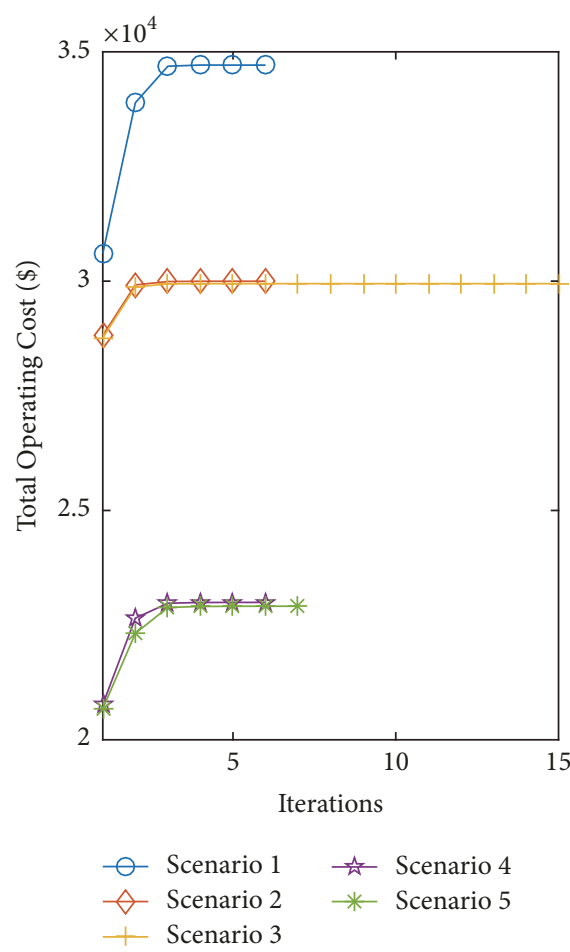

(a)

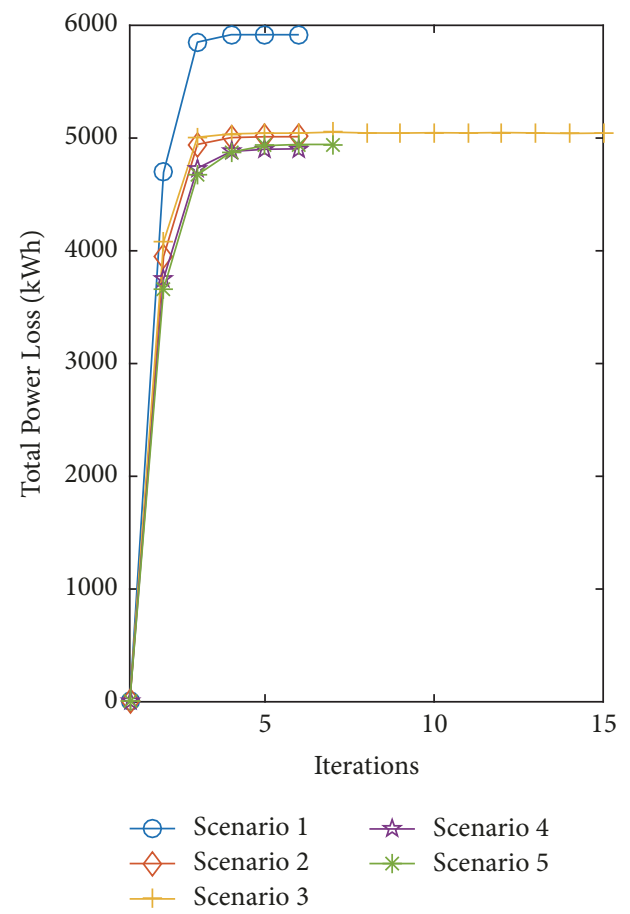

(b)

FIGURE 10: Evolution of (a) total operating cost and (b) total power loss over 24 hours.

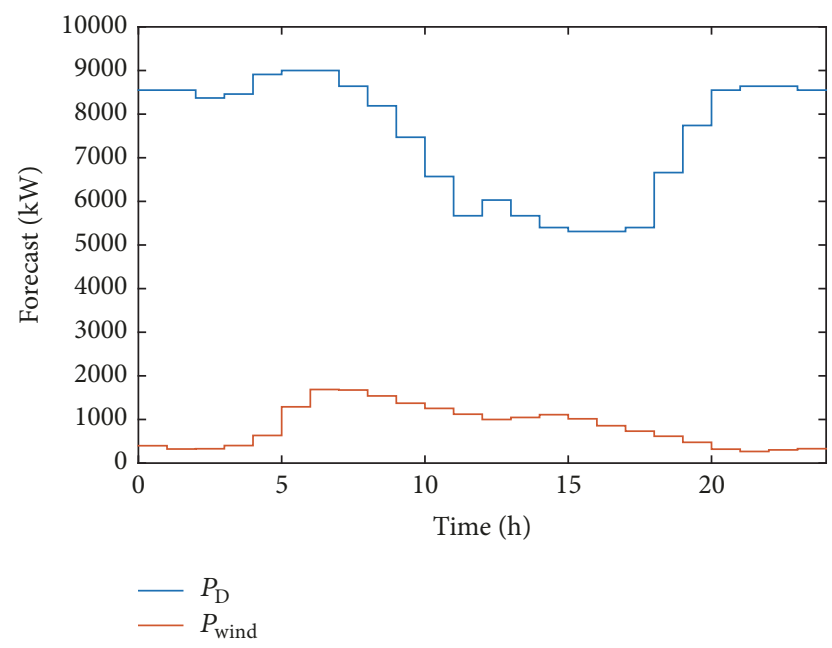

FIGURE 11: Forecasts of load demand and wind power plant generation for Case Study 2.

The concept of eco-industrial parks (EIPs) has been recently gaining research attention. A significant attribute of EIPs is the sharing of energy between different business entities. Typically, industrial parks consume and produce both heat and electricity. In future, the framework proposed in this paper could be integrated with an industrial park level waste heat recovery network with combined cycle gas turbines bridging the electrical and thermal energy streams.
Future studies could also focus on designing efficient load management strategies for such multienergy networks.

\section{Conclusions}

This paper developed a first principle model of industrial MGs including DGs, BESSs, pump loads, and ILs. Subsequently, a 2-stage EMS was proposed for optimally scheduling the MGs. The EMS adopted an iterative procedure to integrate the UC and OPF problems, thereby satisfying network constraints. Load management strategies including pump scheduling and curtailment of ILs were adopted by the EMS to reduce the total electricity cost. The efficacy of the EMS including load management strategies was demonstrated on a 30-bus exemplar MG system under five operational scenarios. From the scheduling results obtained for the 5 scenarios, it was observed that the nonadoption of efficient load management strategies led to significant uncontracted capacity charges. The results also demonstrated the potential of optimal pump scheduling in realizing significant cost savings through the reduction or elimination of uncontracted capacity charges. The impact of auxiliary pumps and curtailment of ILs on the total cost of the system was also analyzed. It was found that while auxiliary pumps had a marginal impact on the total cost, curtailment of ILs realized significant cost savings. Finally, the scalability and efficacy of the EMS were demonstrated on an exemplar 57-bus MG. The 57-bus case study demonstrated the scalability of the EMS architecture 


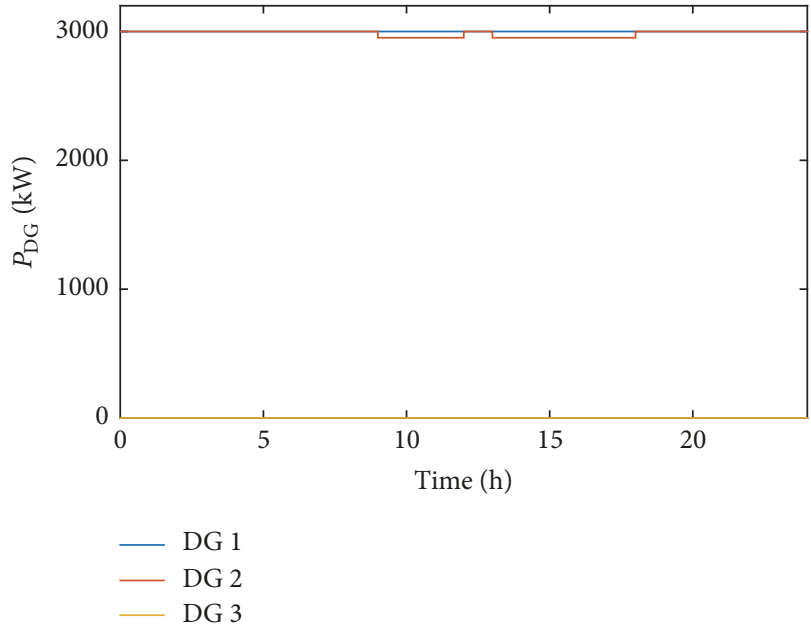

(a)

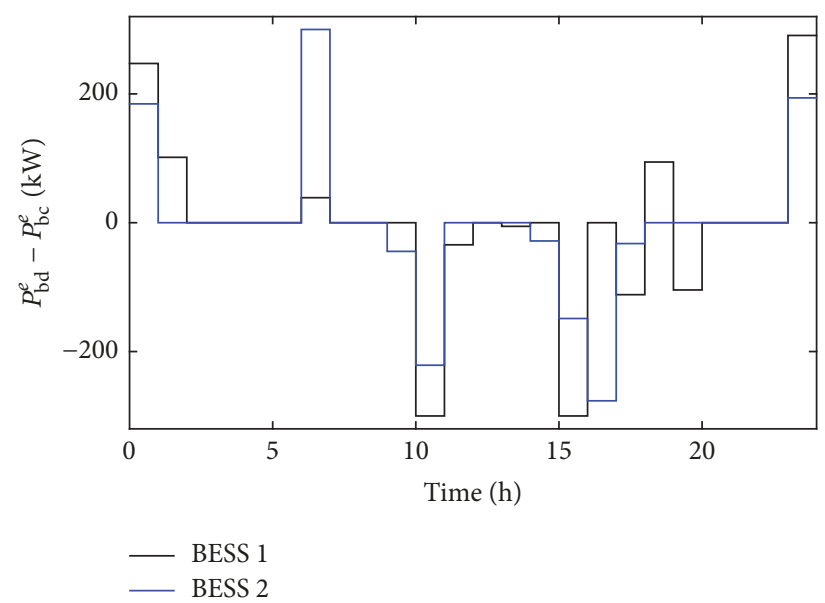

(b)

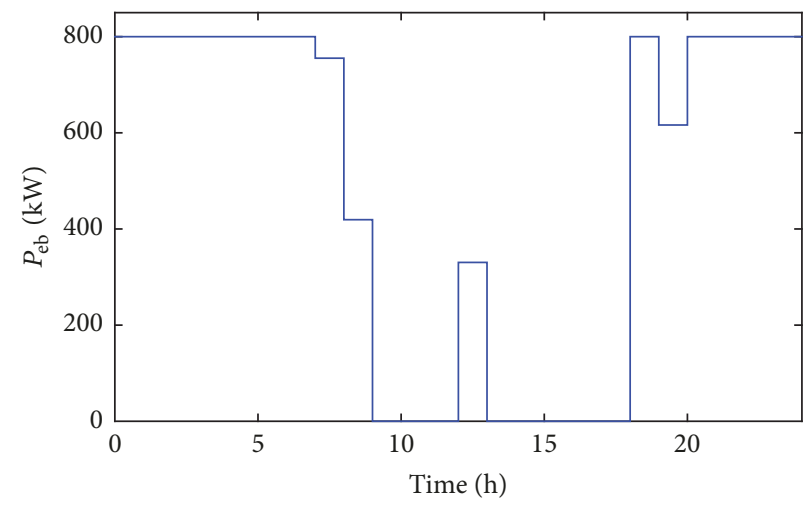

(c)

FIGURE 12: Optimal scheduling of modified IEEE 57-bus system: (a) dispatch values of DG 1, DG 2, and DG 3, (b) charge and discharge profiles of BESSs, and (c) $P_{\mathrm{eb}}$.
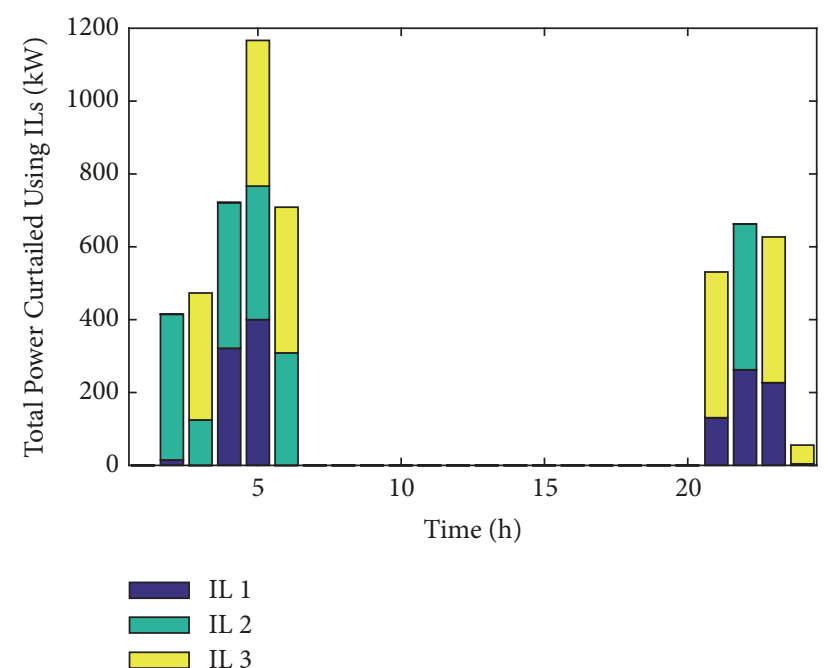

FIGURE 13: Curtailment of ILs in modified IEEE 57-bus system.

while also validating the results obtained in the 30 -bus case study.

\section{Nomenclature}

\section{A. Indices}

$k$ : Index for time (hours)

$f$ : Index for diesel generators (DGs)

$e: \quad$ Index for battery energy storage systems (BESSs)

$h$ : Index for interruptible loads (ILs)

$m$ : Index for pumps

$z: \quad$ Index for renewable energy sources (RESs)

$l: \quad$ Index for lines

$i, j$ : Index for buses.

\section{B. Sets}

$\mathscr{K}$ : Set of all hours in a day; that is, $\mathscr{K}=\{1,2,3, \ldots, 24\}$

$\mathscr{F}$ : Set of DGs in the MG

$\mathscr{E}$ : Set of BESSs in the MG

$\mathscr{H}$ : Set of ILs in the MG

$\mathscr{M}$ : Set of pumps in the MG

$\mathscr{Z}$ : Set of RESs in the MG

$\mathscr{N}$ : Set of buses in the MG

$\mathscr{L}$ : Set of lines in the MG. 


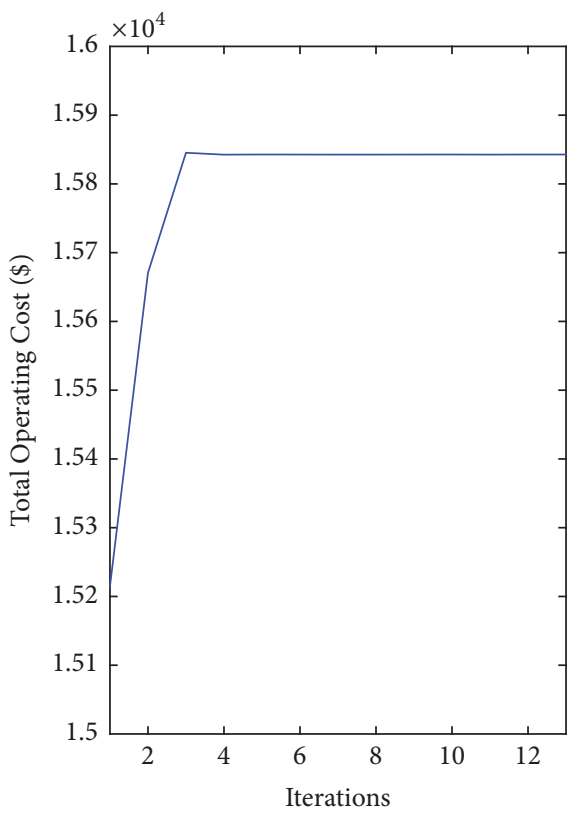

(a)

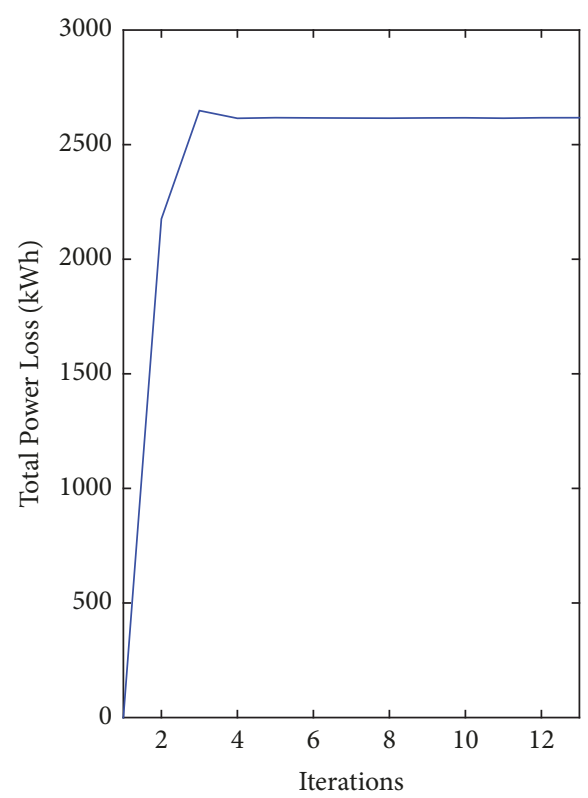

(b)

FIGURE 14: Evolution of (a) total operating cost and (b) total power loss over 24 hours.

\section{Parameters}

$(\cdot)_{\min }$ : Lower bound of the corresponding parameter

$(\cdot)_{\max }: \quad$ Upper bound of the corresponding parameter

$C_{\mathrm{SU}}^{f}: \quad$ DG start-up cost coefficient (\$)

$c_{0}^{f}, c_{1}^{f}, c_{2}^{f}$ : Fuel cost curve coefficients in $\$ / \mathrm{MW}^{2}$, $\$ / \mathrm{MW}$, and \$, respectively

$\eta_{\mathrm{c}}^{e} / \eta_{\mathrm{d}}^{e}$ : $\quad$ BESS charging/discharging efficiency percentages

$P_{1 \mathrm{C}}^{e}$ : $\quad$ Power required by the BESS to charge

$100 \%$ in one hour $(\mathrm{kW})$

$I^{e}: \quad$ BESS purchase cost (\$)

$B_{\text {cap }}^{e}: \quad$ BESS capacity $(\mathrm{kWh})$

$N^{e}$ : $\quad$ Number of cycles for BESS to reach end of life (h)

$T_{\mathrm{bc}}^{e} / T_{\mathrm{bd}}^{e}: \quad$ Average number of hours BESS charges/discharges in a day

$C_{\mathrm{p}}$ : $\quad$ Power coefficient which is a function of the tip speed ratio

$\rho: \quad$ Air density

A: $\quad$ Area swept by the rotor blades

$I_{\mathrm{L}}, I_{\mathrm{S}}$ : Light current and diode saturation current, respectively

$R_{\mathrm{S}}, R_{\mathrm{Sh}}$ : Series and shunt resistances, respectively

$C^{m}: \quad$ Pump capacity in MW

$Q^{m}: \quad$ Pump flow rate in $\mathrm{m}^{3} / \mathrm{h}$

$\tau$ : $\quad$ Optimization interval

$V_{\mathrm{d}}$ : $\quad$ Volume of liquid to be pumped in $24 \mathrm{~h}$ in $\mathrm{m}^{3}$

$P_{\mathrm{CC}}: \quad$ Contracted capacity in MW

$U_{\mathrm{CC}}$ : Uncontracted capacity cost coefficient in \$/MW/month $g_{i j}, b_{i j}:$ Series conductance and susceptance, respectively

$b_{i j}^{\text {sh }}: \quad$ Line charging susceptance.

D. Variables

$b_{\mathrm{SU}, k}^{f}: \quad$ Binary variable indicating DG start-up status

$b_{\mathrm{DG}, k}^{f}: \quad$ Binary variable indicating DG commitment status

$P_{\mathrm{DG}, k}^{f}: \quad$ Real power output of DG $(\mathrm{kW})$

$\mathrm{SOC}_{k}^{e}$ : $\quad$ BESS state of charge (SOC) (scale of 0-1)

$P_{\mathrm{bc}, k}^{e} / P_{\mathrm{bd}, k}^{e}$ : BESS charging/discharging power $(\mathrm{kW})$

$v_{\text {wind }}: \quad$ Wind velocity

$v_{\mathrm{pv}}, i_{\mathrm{pv}}$ : Operating voltage and current of PV module, respectively

$p_{k}^{h}: \quad$ Compensation paid in \$/MWh to IL

$b_{\mathrm{IL}, k}^{h}: \quad$ Binary variable indicating IL status

$P_{\text {IL }, k}^{h}$ : $\quad$ Amount of IL curtailed (kW)

$b_{k}^{m}: \quad$ Binary variable indicating pump status

$b_{\mathrm{SU}, k}^{m}$ : Binary variable indicating pump start-up status

$P_{\mathrm{RES}, k}^{z}$ : Real power produced by RES in $\mathrm{kW}$

$C_{\mathrm{p}, k}: \quad$ Price at which electricity is purchased from the utility grid in $\$ / \mathrm{kWh}$

$P_{\mathrm{eb}, k}: \quad$ Real power purchased from the utility grid in $\mathrm{kW}$

$C_{\mathrm{s}, k}$ : $\quad$ Price at which electricity is sold to the

utility grid in $\$ / \mathrm{kWh}$

$P_{\mathrm{es}, k}: \quad$ Real power sold to the utility grid in $\mathrm{kW}$

$P_{\mathrm{UC}}$ : Uncontracted capacity in MW

$V_{k}^{i}: \quad$ Magnitude of the voltage phasor $v_{k}^{i}$ 
$\delta_{k}^{i}: \quad$ Phase angle of the voltage phasor $v_{k}^{i}$

$s_{k}$ : Complex power injection in MVA

$P_{\mathrm{e}, k}^{i}: \quad$ Generated active power in $\mathrm{kW}$

$Q_{\mathrm{e}, k}^{i}: \quad$ Generated reactive power in kvar

$P_{\mathrm{d}, k}^{i}: \quad$ Active power demand in $\mathrm{kW}$

$Q_{\mathrm{d}, k}^{i}: \quad$ Reactive power demand in kvar

$P_{\mathrm{e}, k}^{i j}: \quad$ Active power flow through line $l$ connecting buses $i$ and $j$ in $\mathrm{kW}$

$Q_{e, k}^{i j}: \quad$ Reactive power flow through line $l$ connecting buses $i$ and $j$ in kvar

$P_{\mathrm{e}, k}^{\text {loss }}: \quad$ Total power loss in $\mathrm{kW}$

$\widehat{P}_{\mathrm{e}, k}^{g}: \quad$ Dispatch value of generator $g$ received by Stage 2 from Stage 1 in $\mathrm{kW}$

$\widehat{u}_{k}^{g}: \quad$ Commitment status of generator $g$ received by Stage 2 from Stage 1

$\widehat{P}_{\mathrm{BESS}, k}^{e}$ : Value of power flow from the BESS received by Stage 2 from Stage 1 in $\mathrm{kW}$.

\section{Conflicts of Interest}

The authors declare that they have no conflicts of interest.

\section{Acknowledgments}

The authors would like to acknowledge funding support from NTU Start-Up Grant. The work of L. P. M. I. Sampath was supported by the International Center of Energy Research (ICER), established by Nanyang Technological University (NTU), Singapore, and Technische Universität München (TUM), Germany.

\section{References}

[1] R. Deng, Z. Yang, M. Chow, and J. Chen, "A survey on demand response in smart grids: mathematical models and approaches," IEEE Transactions on Industrial Informatics, vol. 11, no. 3, pp. 570-582, 2015.

[2] G. Zheng and Q. Huang, "Energy optimization study of rural deep well two-stage water supply pumping station," IEEE Transactions on Control Systems Technology, vol. 24, no. 4, pp. 1308-1316, 2016.

[3] S. Paudyal, C. A. Cañizares, and K. Bhattacharya, "Optimal operation of industrial energy hubs in smart grids," IEEE Transactions on Smart Grid, vol. 6, no. 2, pp. 684-694, 2015.

[4] S. Ashok and R. Banerjee, "An optimization mode for industrial load management," IEEE Transactions on Power Systems, vol. 16, no. 4, pp. 879-884, 2001.

[5] M. P. Fanti, A. M. Mangini, M. Roccotelli, and W. Ukovich, "A district energy management based on thermal comfort satisfaction and real-time power balancing," IEEE Transactions on Automation Science and Engineering, vol. 12, no. 4, pp. 12711284, 2015.

[6] P. Du and N. Lu, "Appliance commitment for household load scheduling," IEEE Transactions on Smart Grid, vol. 2, no. 2, pp. 411-419, 2011.

[7] F. Verrilli, S. Srinivasan, G. Gambino et al., "Model predictive control-based optimal operations of district heating system with thermal energy storage and flexible loads," IEEE Transactions on Automation Science and Engineering, vol. 14, no. 2, pp. 547-557, 2017.

[8] A. Parisio, E. Rikos, and L. Glielmo, "A model predictive control approach to microgrid operation optimization," IEEE Transactions on Control Systems Technology, vol. 22, no. 5, pp. 1813-1827, 2014.

[9] A. Parisio, E. Rikos, G. Tzamalis, and L. Glielmo, "Use of model predictive control for experimental microgrid optimization," Applied Energy, vol. 115, pp. 37-46, 2014.

[10] A. Krishnany, B. V. Patilx, H. B. Gooiy, and K. V. Lingy, "Predictive control based framework for optimal scheduling of combined cycle gas turbines," in Proceedings of the 2016 American Control Conference, (ACC '16), pp. 6066-6072, USA, July 2016.

[11] Y. Fu, M. Shahidehpour, and Z. Li, "Security-constrained unit commitment with ac constraints," IEEE Transactions on Power Systems, vol. 20, no. 3, pp. 1538-1550, 2005.

[12] M. Kahl, C. Freye, and T. Leibfried, "A Cooperative MultiArea Optimization with Renewable Generation and Storage Devices," IEEE Transactions on Power Systems, vol. 30, no. 5, pp. 2386-2395, 2015.

[13] P. Fortenbacher, A. Ulbig, S. Koch, and G. Andersson, "Gridconstrained optimal predictive power dispatch in large multilevel power systems with renewable energy sources, and storage devices," in Proceedings of the 2014 IEEE PES Innovative Smart Grid Technologies Conference Europe, ISGT-Europe 2014, Turkey, October 2014.

[14] A. Ouammi, H. Dagdougui, L. Dessaint, and R. Sacile, "Coordinated Model Predictive-Based Power Flows Control in a Cooperative Network of Smart Microgrids," IEEE Transactions on Smart Grid, vol. 6, no. 5, pp. 2233-2244, 2015.

[15] S. X. Chen and H. B. Gooi, "Jump and shift method for multi-objective optimization," IEEE Transactions on Industrial Electronics, vol. 58, no. 10, pp. 4538-4548, 2011.

[16] L. P. Sampath, A. Krishnan, K. Chaudhari, H. B. Gooi, and A. Ukil, "A control architecture for optimal power sharing among interconnected microgrids," in Proceedings of the 2017 IEEE Power \& Energy Society General Meeting (PESGM), pp. 1-5, Chicago, IL, July 2017.

[17] K. Chaudhari, A. Ukil, K. N. Kumar, U. Manandhar, and S. K. Kollimalla, "Hybrid Optimization for Economic Deployment of ESS in PV-Integrated EV Charging Stations," IEEE Transactions on Industrial Informatics, vol. 14, no. 1, pp. 106-116, 2018.

[18] Z. Bao, Q. Zhou, Z. Yang, Q. Yang, L. Xu, and T. Wu, "A multi time-scale and multi energy-type coordinated microgrid scheduling solution - Part I: model and methodology," IEEE Transactions on Power Systems, vol. 30, no. 5, pp. 2257-2266, 2015.

[19] Z. Li and Y. Xu, "Optimal coordinated energy dispatch of a multi-energy microgrid in grid-connected and islanded modes," Applied Energy, vol. 210, pp. 974-986, 2018.

[20] C. Grigg, P. Wong, P. Albrecht, R. Allan, M. Bhavaraju, and R. Billinton, "The IEEE reliability test system -1996 a report prepared by the reliability test system task force of the application of probability methods subcommittee," IEEE Transactions on Power Systems, vol. 14, no. 3, pp. 1010-1020, 1999.

[21] W. de Soto, S. A. Klein, and W. A. Beckman, "Improvement and validation of a model for photovoltaic array performance," Solar Energy, vol. 80, no. 1, pp. 78-88, 2006. 
[22] X. Wang, A. Palazoglu, and N. H. El-Farra, "Operational optimization and demand response of hybrid renewable energy systems," Applied Energy, vol. 143, pp. 324-335, 2015.

[23] "Renewables.ninja," https://www.renewables.ninja/.

[24] M. Alipour, K. Zare, and M. Abapour, "MINLP Probabilistic Scheduling Model for Demand Response Programs Integrated Energy Hubs," IEEE Transactions on Industrial Informatics, vol. 14, no. 1, pp. 79-88, 2017.

[25] A. Krishnan, L. P. M. I. Sampath, F. Y. S. Eddy, and B. V. Patil, "Multi-energy scheduling using a hybrid systems approach," in Analysis and Design of Hybrid Systems, 2018, https://www .researchgate.net/publication/323905991.

[26] C. A. Floudas, Nonlinear and Mixed-Integer Optimization: Fundamentals and Applications, Oxford University Press, NY, USA, 1995.

[27] A. Krishnan, F. Y. S. Eddy, and B. V. Patil, "Hybrid Model Predictive Control Framework for the Thermal Unit Commitment Problem including Start-up and Shutdown Power Trajectories," IFAC-PapersOnLine, vol. 50, no. 1, pp. 9329-9335, 2017.

[28] F. D. Torrisi and A. Bemporad, "HYSDEL-A tool for generating computational hybrid models for analysis and synthesis problems," IEEE Transactions on Control Systems Technology, vol. 12, no. 2, pp. 235-249, 2004.

[29] A. Bemporad and M. Morari, "Control of systems integrating logic, dynamics, and constraints," Automatica, vol. 35, no. 3, pp. 407-427, 1999.

[30] J. Löfberg, "YALMIP: a toolbox for modeling and optimization in MATLAB," in Proceedings of the IEEE International Symposium on Computer Aided Control System Design, pp. 284-289, Taipei, Taiwan, September 2004.

[31] M. Q. Wang, H. B. Gooi, S. X. Chen, and S. Lu, "A mixed integer quadratic programming for dynamic economic dispatch with valve point effect," IEEE Transactions on Power Systems, vol. 29, no. 5, pp. 2097-2106, 2014.

[32] F. Capitanescu and L. Wehenkel, "Experiments with the interior-point method for solving large scale Optimal Power Flow problems," Electric Power Systems Research, vol. 95, pp. 276-283, 2013.

[33] E. J. Oliveira, L. W. Oliveira, J. Pereira, L. M. Hon, I. C. Silva, and A. Marcato, "An optimal power flow based on safety barrier interior point method," International Journal of Electrical Power \& Energy Systems, vol. 64, pp. 977-985, 2015.

[34] R. D. Zimmerman, C. E. Murillo-Sánchez, and R. J. Thomas, "MATPOWER: steady-state operations, planning, and analysis tools for power systems research and education," IEEE Transactions on Power Systems, vol. 26, no. 1, pp. 12-19, 2011.

[35] "Energy market company, price information," https://www .emcsg.com/marketdata/priceinformation.

[36] X. Zhang, J. Bao, R. Wang, C. Zheng, and M. Skyllas-Kazacos, "Dissipativity based distributed economic model predictive control for residential microgrids with renewable energy generation and battery energy storage," Journal of Renewable Energy, vol. 100, pp. 18-34, 2017. 


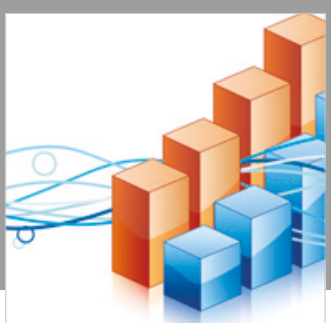

Advances in

Operations Research

\section{-n-m}
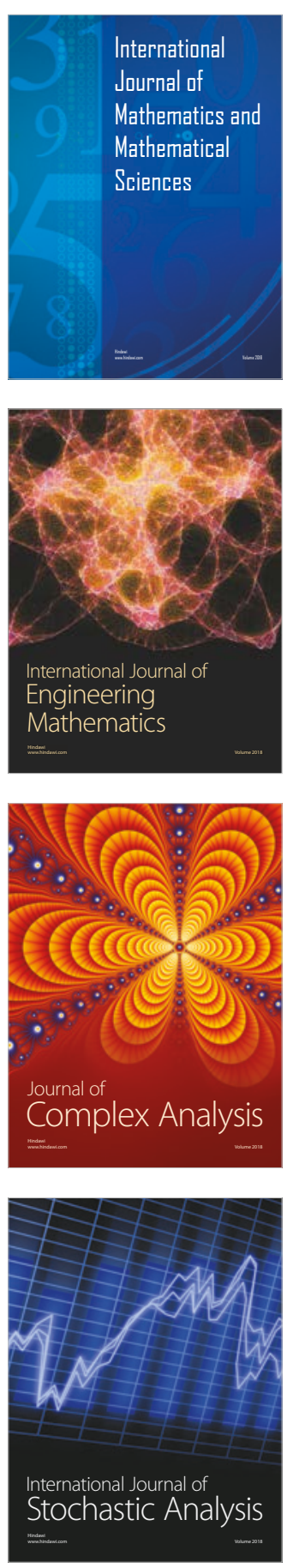
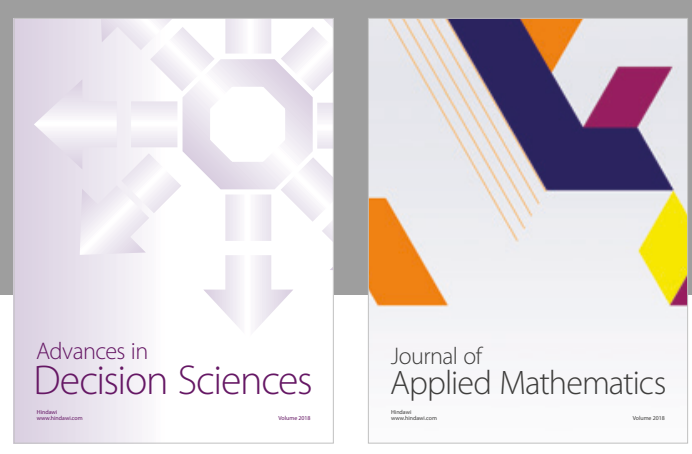

Journal of

Applied Mathematics
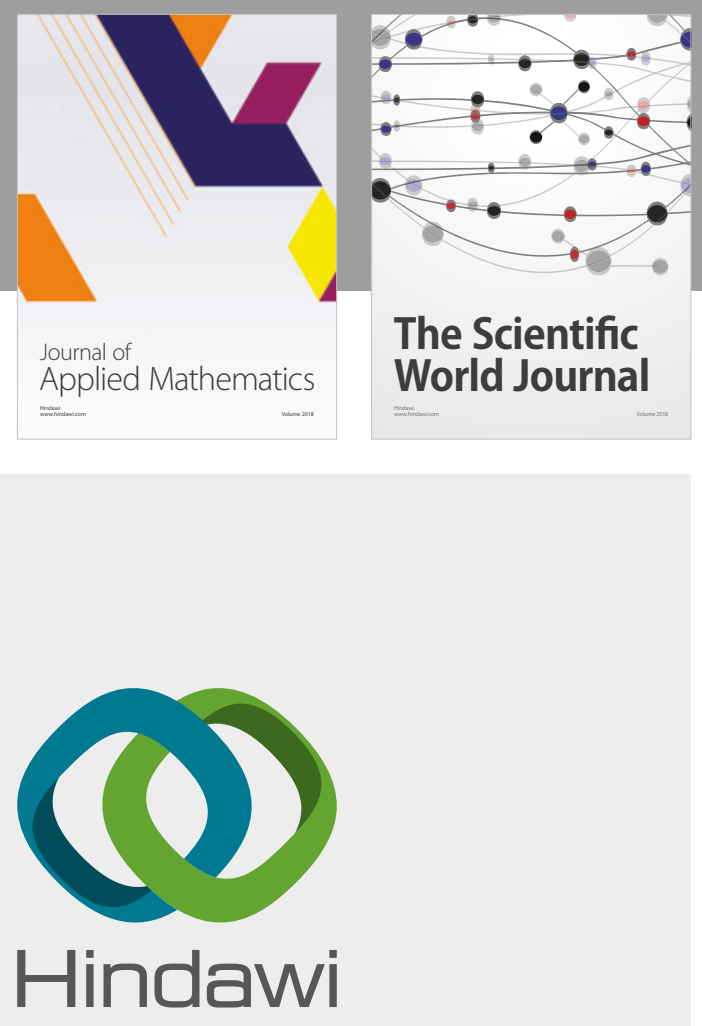

Submit your manuscripts at

www.hindawi.com

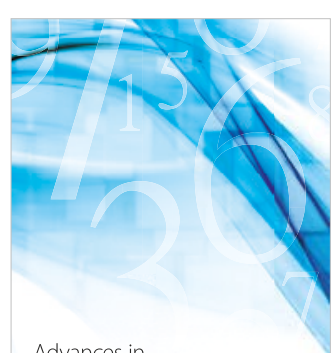

Advances in
Numerical Analysis
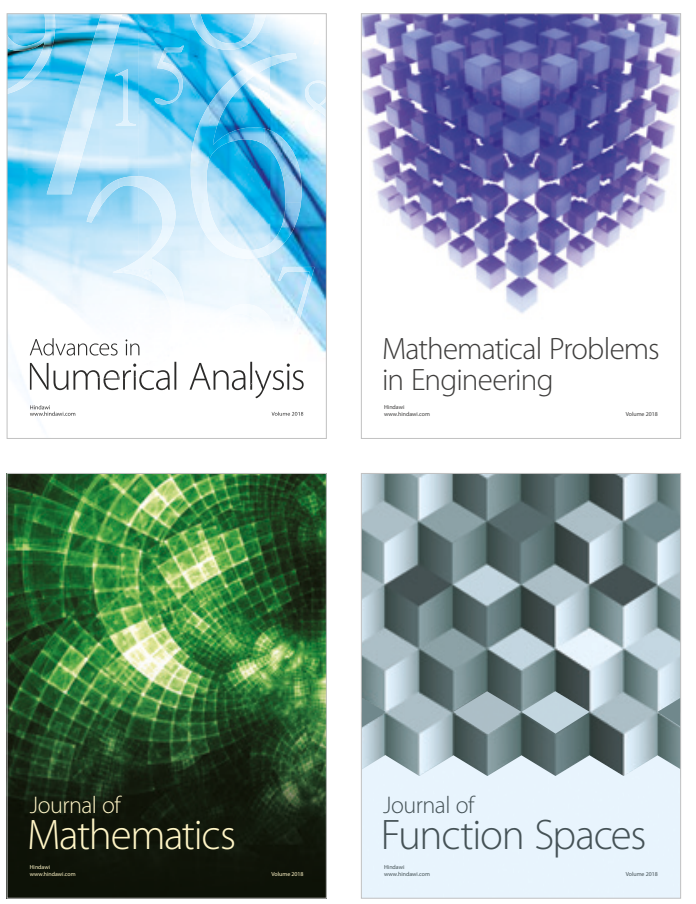

Mathematical Problems in Engineering

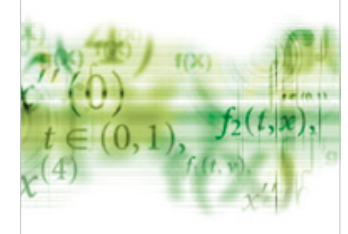

International Journal of

Differential Equations

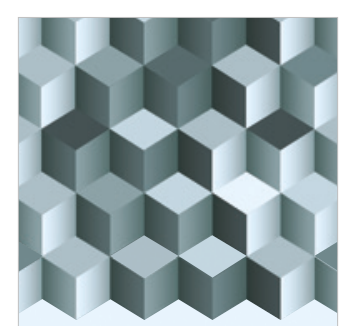

Journal of

Function Spaces
The Scientific

World Journal

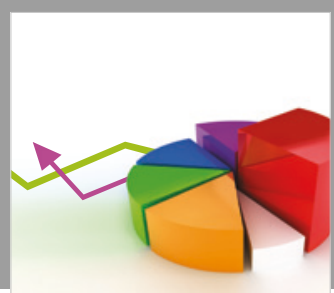

Journal of

Probability and Statistics
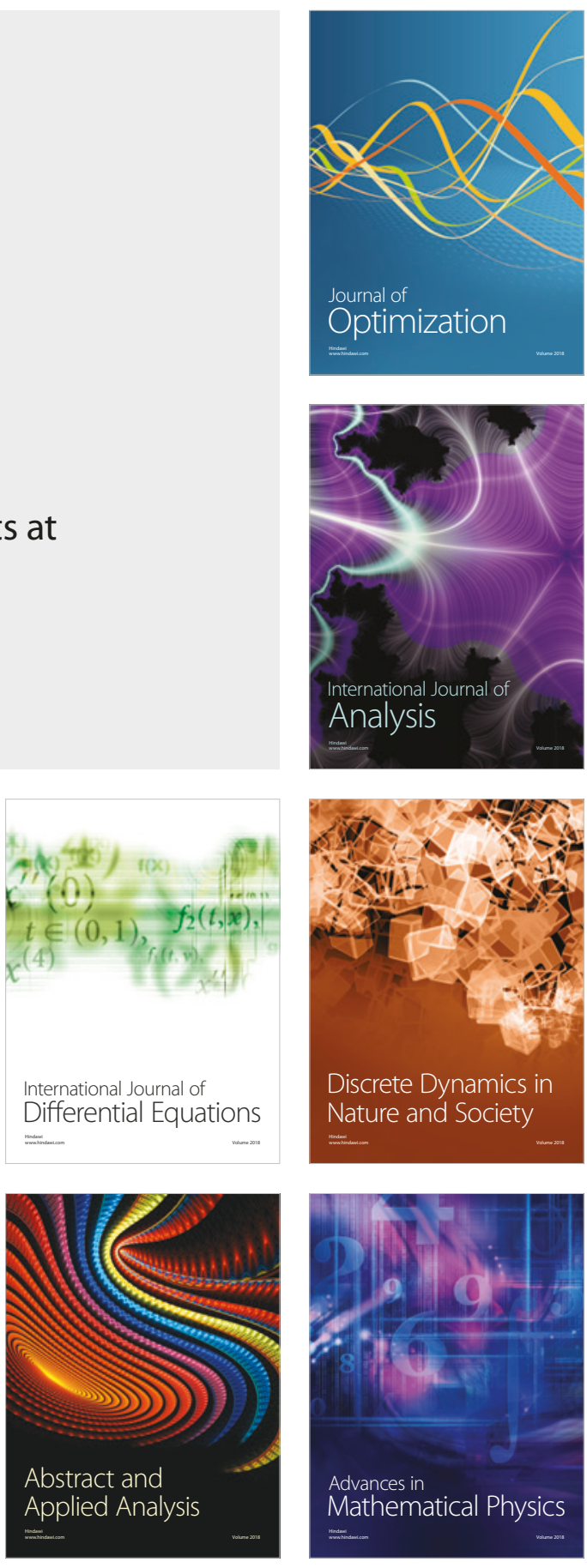